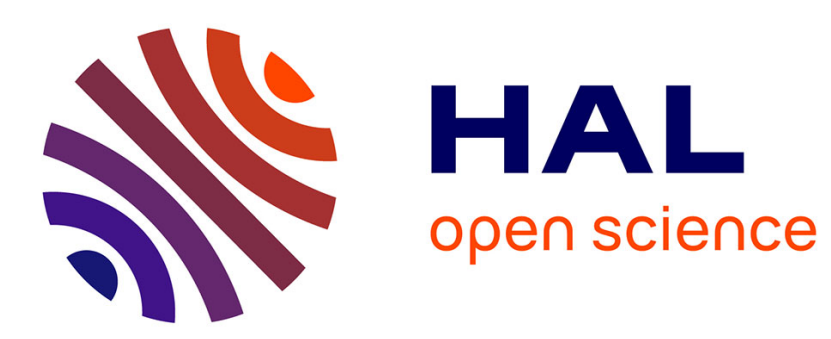

\title{
Effective site energy and cluster expansion approaches for the study of phase diagrams
}

F Berthier, Q Lullien, B Legrand

\section{To cite this version:}

F Berthier, Q Lullien, B Legrand. Effective site energy and cluster expansion approaches for the study of phase diagrams. Physical Review B, 2021, 104, 10.1103/physrevb.104.014111 . hal-03305996

\section{HAL Id: hal-03305996 \\ https://hal.science/hal-03305996}

Submitted on 28 Jul 2021

HAL is a multi-disciplinary open access archive for the deposit and dissemination of scientific research documents, whether they are published or not. The documents may come from teaching and research institutions in France or abroad, or from public or private research centers.
L'archive ouverte pluridisciplinaire HAL, est destinée au dépôt et à la diffusion de documents scientifiques de niveau recherche, publiés ou non, émanant des établissements d'enseignement et de recherche français ou étrangers, des laboratoires publics ou privés. 


\title{
Effective site energy and cluster expansion approaches for the study of phase diagrams
}

\author{
F. Berthier $\odot,{ }^{1},{ }^{*}$ Q. Lullien, ${ }^{1}$ and B. Legrand ${ }^{2}$ \\ ${ }^{1}$ Université Paris-Saclay, CNRS, Institut de chimie moléculaire et des matériaux d'Orsay, 91405 Orsay, France \\ ${ }^{2}$ Université Paris-Saclay, CEA, Service de Recherches de Métallurgie Physique, 91191 Gif-sur-Yvette, France
}

(Received 5 May 2021; revised 8 July 2021; accepted 8 July 2021; published 22 July 2021)

\begin{abstract}
We apply the cluster expansion (CE) method to determine the effective cluster interactions (ECIs) from a simple energetic model that depends on both local and global composition. This model is defined by the site energies of random solid solutions of a one-dimensional alloy Co-Pt. We explore how these local and global dependencies are reflected on the cluster interactions. The energies of the structures are not well reproduced with concentration-independent interactions. Moreover, the interactions have a larger range than the energetic model which is limited to the nearest neighbors. By fitting the ECIs on the site energies, we suggest a mean-field-type weighting of the excess variables present in clusters of large size. We show that the site energy formalism controls the size of the clusters required for CE convergence and their concentration dependence. Finally, we take advantage of the site energy formalism to describe the elastic and chemical effects that control the thermodynamics of the alloy as a function of the ECIs.
\end{abstract}

DOI: 10.1103/PhysRevB.104.014111

\section{INTRODUCTION}

The determination of phase diagrams is a preliminary step which is essential for the study of alloys. A first method consists of describing alloys from a generalized Ising model based on effective cluster interactions (ECIs) [1-5]. The cluster interactions are determined from the energy of many ordered structures issued from $a b$ initio calculations [6,7]. This approach is known as cluster expansion (CE). A second method consists of the definition of interatomic potentials to perform atomic simulations with relaxations. However, simulations with semi-empirical interatomic potentials have two drawbacks: the reliability of these potentials and their lack of transparency regarding the physics they contain.

CE has already been used to construct interatomic potentials [8-10]. In the same spirit, we propose to determine the effective interactions between clusters contained in interatomic potentials, thanks to the $\mathrm{CE}$ method, to have a better understanding of these potentials and then to improve them. Among the known interatomic potentials, we use N-body interatomic potentials derived from the second-moment approximation (SMA) of the tight-binding scheme $[11,12]$. It has been shown that these potentials are relevant for both bulk and surface studies [13-17]. N-body interatomic potentials lead to a good description of surface relaxations as compared with pair potentials and are therefore well suited for surface segregation studies. Although very efficient, these potentials do not allow simultaneous adjustment of the order/disorder or demixing/disorder critical temperature of the system and the solution energies of dilute systems [18]. The analysis of the ECIs obtained by CE from $a b$ initio calculations and by SMA potentials should lead to the determination of the interactions

*fabienne.berthier@universite-paris-saclay.fr that are missing in these potentials and that are quantifiable only by advanced analyses such as the one we propose.

$\mathrm{CE}$ is a powerful tool for the analysis of alloy thermodynamics and is widely used to study bimetallic alloys [19-26], high entropy alloys [27], semiconductors, and oxides [28-33]. However, its application to real systems is often questionable and remains a topical issue even before considering the difficulties associated with complex structures such as inhomogeneous systems. CE consists of fitting the values of cluster interactions of a rigid-lattice Hamiltonian from the configurational energies of a set of ordered structures. Its realization requires different steps beforehand such as choosing the number of structures and the structures themselves and choosing the range and type of cluster interactions (pairs, triplets, ...n-tuples). Once the ECIs are estimated by minimization, the predictive ability is then tested on a set of new configurations, and the procedure is repeated by increasing the range of cluster interactions until satisfactory prediction quality is obtained. The accuracy of CE predictions depends on all these choices. Since the initial founding papers, algorithms have been developed [19,34,35] and several improvements and efficient methods have been proposed to assess the quality of the fit and to select clusters [36-43]. Note, for example, that the cluster interactions are generally independent of concentration, while the configurations that allow one to determine them have different compositions. The implementation of $\mathrm{CE}$ with concentration-dependent interactions enables us to consider volume variations linked to composition variations. Despite concentration-dependent CE converges much faster and is more accurate, only a few studies address concentration-dependent interactions [36,37,39,44,45].

We propose a detailed CE analysis using the effective site energies (ESEs) description [46-48]. Let us recall the principles of this approach. The energies of each atom of random solid solutions (RSSs) are determined and sorted according 
to both the local composition (restricted to first neighbors) and the nominal concentration. RSSs are relaxed by molecular static simulations using SMA interatomic potentials. These site energies are averaged and introduced in a rigid-lattice Hamiltonian. Therefore, the Hamiltonian, which is a combinatorial summation of the ESEs, depends both on the local composition and on the nominal concentration. This description allowed us to highlight the role of first-neighborhood triplet interactions in thermodynamic properties [47].

In this paper, we propose a theoretical study using an energetic model with a known range of interactions. This energetic model is a simplified expression of site energies calculated from a one-dimensional (1D) $\mathrm{Co}_{c} \mathrm{Pt}_{1-c}$ system. The great advantage is to know the range of the interactions contained in this energetic model since its dependency with local environment is limited to the nearest-neighboring shell. This poses a problem rarely addressed so far in the literature of the introduction into CE via the ECIs of a phantom dependency with the local environment, i.e., with further neighbors than those considered in the initial model. These clusters couple configuration variables that are not in the original model and that need to be treated in a specific way.

The analysis is then extended to ECIs which are fitted on the site energies instead of the energy of a set of ordered structures. The site energies fix the number of equations to be considered to fit the interactions, unlike the fit on the structures. The energies of site, and thus the ECIs, allow one to decompose the mixing and permutation enthalpies into chemical contribution [related to local effects that drive short-range order (SRO)] and elastic contribution (related to the variation of the volume with the concentration). This analysis gives a physical content to Calphad-type empirical methods $[49,50]$.

This paper is organized as follows. The energetic model is introduced in Sec. II A, followed by the CE approach in Sec. II B 1. Results obtained with concentration-independent and concentration-dependent interactions are presented in Sec. II B 2. Section III is devoted to the development of $\mathrm{CE}$ from the site energies. We first present the formalism (Sec. III A ) and then the results (Sec. III B) with concentration-independent (Sec. III B 1) and concentrationdependent interactions (Sec. III B 2), followed by a discussion in Sec. III B 3. The analysis of the mixing and permutation enthalpies in terms of elastic and chemical contributions is then described in Sec. IV. After a discussion about the possible extensions of the approach (SEC. V), we conclude in Sec. VI.

\section{ECIs FROM CE}

\section{A. Energetic model}

To focus the discussion on the coherence between the range of interactions of the energetic model and the length of $n$-tuples involved in CE, the energetic model is expressed in terms of site energies whose dependency range with the local composition can be chosen. Site energies are obtained from RSSs by molecular static simulations using interatomic potentials [46-48]. The site energy is the average of all energies of sites which are occupied by an atom of the same type and which have the same local environment. For an $A_{c} B_{1-c}$ alloy, the site energies therefore depend both upon the local composition and the nominal concentration. The local environment is characterized by the numbers $p_{1}, p_{2}, \ldots p_{i}$ of $A$ atoms (but it would also be $B$ ) in the first, second, ... $i$ th-neighboring shells. For the sake of simplicity, the length of interactions is restricted hereafter to the first-neighboring shell, and we consider a 1D system. If this simplification has a theoretical motivation, 1D alloys can occur along the steps of a surface, the edges of a cluster, or in the core of dislocation. The site energies are written $E_{I}^{p}$ with $I=A$ or $B$ and $p=p_{1}$. For a 1D system, $p$ varies from 0 to 2 . We have determined the ESEs of a $\mathrm{Co}_{c} \mathrm{Pt}_{1-c}$ linear chain to define our energetic model using interatomic potentials already considered in the literature for their abilities to reproduce bulk and surfaces properties [51,52]. CoPt is a well-known alloy, which has often been studied both in bulk and in two-dimensions (2D) [26,53-55]; this paper is an extension to 1D. Note that the purpose of this paper is not the alloy itself. The alloy is only used to define the energetic model. Moreover, both the SMA and the analytical model derived from it cannot include the contributions from configurational effects of partial order on the electronic structure [56]. This energetic model is simple but raises important questions that are not often stated and helps to capture general trends.

These energies can be written in a simplified way as

$$
\begin{aligned}
E_{A}^{p}(c)= & -3.24-0.74 c+0.8 c^{2} \\
& +\frac{1}{2}\left(0.8+0.4 c-0.87 c^{2}\right) p \\
& +\left(-0.07-0.04 c^{2}\right)(2-p) p, \\
E_{B}^{p}(c)= & -3.67+\frac{1}{2}(0.26) p .
\end{aligned}
$$

The dependence on concentration of site energies is illustrated in Fig. 1 for (a) $A$ atoms and (b) $B$ atoms and for $p=0$, 1 , or 2. Here, $E_{A}^{p}$ is a second-order function in $c$, while $E_{B}^{p}$ is concentration independent.

These site energies are expressed as the sum of a main linear term in $p$ and a complementary quadratic term in

$$
E_{I}^{p}(c)=E_{I}^{0}(c)+\frac{p}{Z}\left[E_{I}^{Z}(c)-E_{I}^{0}(c)\right]+\varkappa_{I}(c) p(Z-p),
$$

with $Z$ the coordination number $(Z=2$ at $1 \mathrm{D})$ and $\varkappa_{1}(c)$ the curvatures with $I=A$ or $B$. In the present case, $\varkappa_{B}(c)=0 \forall c$. The curvature is nonzero for $A$. At $1 \mathrm{D}$, the curvature occurs only for $p=1$.

The matching between the site energies and a generalized Ising model allows one to relate the linear term to pair interactions. The complementary quadratic term, which represents the excess energy as compared with pair interactions, corresponds to the contribution of triplets [47]. Contrary to a generalized Ising model, pair and triplet interactions depend on the nominal concentration $c$.

This model clearly separates the dependence with the local chemical composition $p$ and the nominal composition $c$. This allows us to easily separate the elastic effects (related to a variation of site energies with concentration at a given local composition) from the chemical effects (related to a variation of site energies with local composition at a given nominal concentration). This point is detailed in Appendix A. 

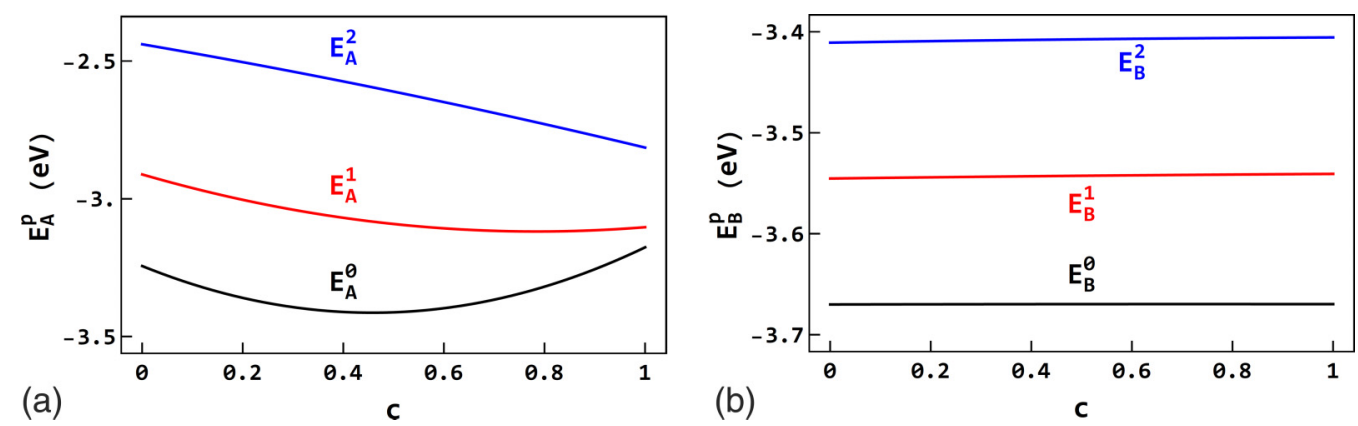

FIG. 1. Site energy of an atom (a) $A$ and (b) $B$ as a function of the nominal concentration $c$ in $A_{c} B_{1-c}$ alloy for a number $p$ of $A$ neighbors equals to 0 (black), 1 (red), and 2 (blue) with $A=$ Co and $B=\mathrm{Pt}$.

\section{B. CE method}

\section{Formalism}

$\mathrm{CE}$ is based on the calculation of the energy of many ordered phases at different concentrations to find the best ECI values to describe the energies accurately. The energy dataset is most often obtained by ab initio calculations, but we can also get it by using interatomic potentials adjusted from experimental data or from $a b$ initio calculations. In this paper, instead of $a b$ initio calculations, we use the energetic model defined by Eq. (1) to calculate the energy of a given configuration.

In a rigid-lattice approximation, the total energy of a linear chain with periodic boundary conditions for an $A_{c} B_{1-c}$ alloy is then given by

$$
E=\sum_{j=1}^{N} q_{j}^{I} E_{I}^{q_{j-1}^{A}+q_{j+1}^{A}}(c)
$$

where $q_{j}^{I}$ is the occupation number which equals 1 if the site $j$ is occupied by an atom $I$, and 0 otherwise. The local composition $p$ depends on the occupation number of the two adjacent sites ( $p=q_{j-1}^{A}+q_{j+1}^{A}$ ). Here, $N$ is the total number of sites.

By setting $q_{j}=q_{j}^{A}$, Eq. (3) becomes

$$
E=\sum_{j=1}^{N}\left[q_{j} E_{A}^{q_{j-1}+q_{j+1}}(c)+\left(1-q_{j}\right) E_{B}^{q_{j-1}+q_{j+1}}(c)\right] .
$$

Equation (4) gives the energy of a configuration that we subsequently use as the basis for ECI calculations.

The energy of a configuration can also be described using a Hamiltonian written as a linear combination of $n$-tuple interactions:

$H=\frac{1}{2 !} \sum_{I J} \sum_{l, m \neq l} q_{l}^{I} q_{m}^{J} V_{l m}^{I J}+\frac{1}{3 !} \sum_{I J K} \sum_{l, m n m} q_{l}^{I} q_{m}^{J} q_{n}^{K} V_{l m n}^{I J K}+\ldots$,

where the expansion coefficients $V_{l m}^{I J}, V_{l m n}^{I J K}, \ldots$ are the ECIs of pairs, triplets, ... to be identified. At $1 \mathrm{D}$, an $n$-tuple is a chain of $n$ successive atoms; it has a unique form and depends on its length and on the type of atoms.

In practice, the Hamiltonian is truncated because it is not possible to consider all the clusters, but it must remain predictive. The main task of the CE method is to determine the preponderant ECIs by least square fitting of the total energy of a set of structures (called the training set). A truncation of the summation of the Hamiltonian to pair interactions yields the Ising model. The short-range interactions as pairs, triplets, and quadruplets are generally preponderant ECIs. To measure the accuracy of the fit, we calculate the root mean square error (RMSE) of the predictions for all configurations. The ability to reproduce the system is then tested on new structures (named the test set).

As stated previously in the introduction, in most of this literature, ECIs are calculated in the grand-canonical ensemble and so are independent of nominal concentration, while the composition of the structures varies. Asta et al. [36] and Wolverton et al. [37] have shown that CE is more accurate in the canonical ensemble with concentration-dependent ECIs. The two cases are presented below.

\section{Results}

We consider 33 concentration values between 0 and 1 . In total, the set of structures is composed of 355 chains of 120 sites with periodic boundary conditions. For each concentration, 10 configurations are randomly generated. We add the 25 possible ordered configurations for this chain length.

First, we determine concentration-independent ECIs. The Hamiltonian summation is successively restricted to pairs, triplets, ... until the sextuples. The mixing enthalpy $\Delta H^{\operatorname{mix}}(c)=H(c)-\left[c E_{A}^{Z}(c=1)+(1-c) E_{B}^{0}(c=0)\right], \quad$ calculated from Eq. (5) with ECIs issued from the CE, is compared with the direct mixing enthalpy given by Eq. (4). Figure 2(a) shows a weak agreement between the two quantities. The fitting error, quantified by the RMSE, decreases slowly as the length of the $n$-tuple increases [Fig. 2(b)]. This result confirms that, when the energy of formation of random alloy depends quadratically on the concentration, the decay of the root mean square is slow [45,57].

We then investigated concentration-dependent ECIs from the same set of training configurations. To capture the concentration dependence without searching for long-range tuples, it is necessary to implement concentration-based CE to obtain ECIs for each nominal concentration. Then the agreement is excellent with only pairs and triplets (Fig. 3). CE really converges faster with concentration-dependent ECIs than with concentration-independent ECIs, and the fitting error is much 

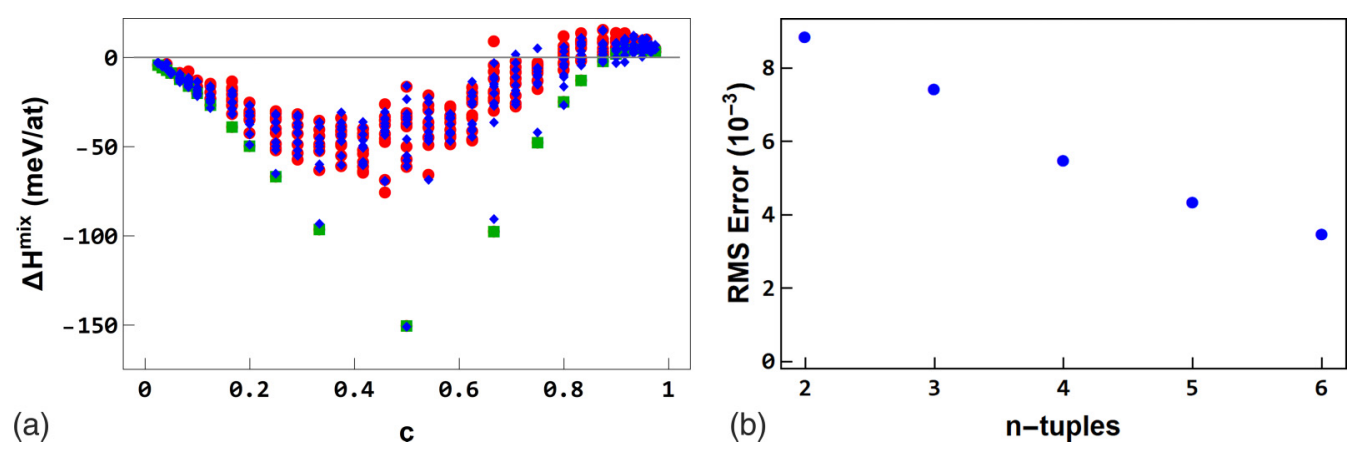

FIG. 2. (a) Evolution as a function of the concentration $c$ of $\Delta H^{\text {mix }}$ calculated from the energetic model (random solid configurations: red points, ordered configurations: green squares) and with concentration-independent effective cluster interactions (ECIs) until sextuples issued from the cluster expansion (CE; blue diamonds). (b) Evolution of the root-mean-square error as a function of the length of $n$-tuples.

smaller $\sim 10^{-13}$ (see Table I). This result confirms those of the literature $[25,39,44,45,57]$.

As can be seen in Fig. 4, the ECIs depend strongly on the concentration. The heteroatomic pairs [in Fig. 4(a)] are equal $\left(V_{A B}=V_{B A}\right)$ on the whole range of concentration. Symmetrical relationships are also observed [Fig. 4(d)] for triplets $\left(V_{A B B}=V_{B B A}, V_{A A B}=V_{B A A}\right)$.

This raises the question of whether these ECIs can reproduce the original energetic model. We therefore calculate the energies of each site for each structure from the ECIs and deduce the average site energies as a function of the concentration and of the first-neighboring local environment. We also calculate the site energies without using the structures, but by considering the value of $p$ which characterizes the local chemical composition of the two nearest neighbors. For example, the site energy of an atom $A$ surrounded by two atoms $A$ (see Fig. 5) $E_{A}^{2}$ is easily written according to the pair interactions $E_{A}^{2}=\frac{1}{2}\left(2 V_{A A}\right)$. With triplet interactions, it is less obvious since the central atom $A$ belongs to three triplets, and atoms of the second-neighboring shell are involved for two of them. The chemical nature of these atoms is unknown since the site energies are limited to the

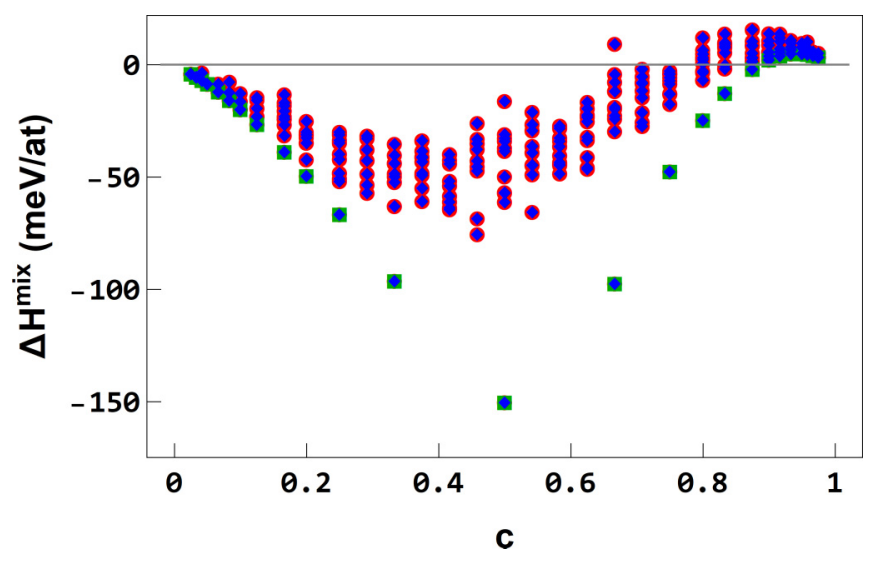

FIG. 3. Evolution as a function of the concentration $c$ of $\Delta H^{\text {mix }}$ calculated from the energetic model (random solid configurations: red points, ordered configurations: green squares) and with concentration-dependent effective cluster interactions (ECIs) until triplets issued from the cluster expansion (CE; blue diamonds). first neighbors. The site energy is thus $\frac{1}{3}\left(V_{I A A}+V_{A A A}+V_{A A J}\right)$ with $I, J=A$ or $B$, and four values are possible. The two methods give consistent results but are far from the energetic model (Fig. 6).

Thus, although CE leads to a very good fit of the total energies, the ECIs do not reproduce the site energies that are contained in the total energies. This result shows that two different energetic models can lead to identical structure energies.

\section{DETERMINATION OF ECIS FROM SITE ENERGIES}

\section{A. Formalism}

Instead of fitting as best as possible the ECIs from the total energy of several structures, we propose to determine them from the site energies. If ECIs reproduce the site energies, then they should also reproduce the energies of the structures. We therefore first optimize the ECIs starting from the site energy functions and then crosscheck these fittings on the configurations. While the number of structures needed to accurately determine ECIs from the total energy is unknown, the number of site energies to be fitted is well known. Thus, for a $1 \mathrm{D}$ system with $Z=2$, the ECIs are identified through six relations $\left[E_{I}^{p}(c)\right.$ with $I=A$ or $B$ and $p=0,1$, or 2]. This approach allows one to control the conditions necessary for a CE.

Another great advantage of this approach is to highlight the problem of inconsistency between the length of the $n$ tuples and the range of the energetic model. When the size of the $n$-tuples is greater than the range of the site energies,

TABLE I. Comparison of root mean square for the different approximations of $\mathrm{CE}$ as a function of the tuple length for the 355 structures in the training set. CE of structures (a) and (b) and of site energies (c) and (d) with concentration-independent ECIs (a) and (c) and concentration dependent ECIs (b) and (d).

\begin{tabular}{lccccc}
\hline Method & $n=2$ & $n=3$ & $n=4$ & $n=5$ & $n=6$ \\
\hline (a) & $8.810^{-3}$ & $7.410^{-3}$ & $5.510^{-3}$ & $4.310^{-3}$ & $3.510^{-3}$ \\
(b) & $2.210^{-3}$ & $8.410^{-14}$ & & & \\
(c) & $7.010^{-2}$ & $2.010^{-2}$ & $1.710^{-2}$ & $1.110^{-3}$ & $1.410^{-5}$ \\
(d) & $1.610^{-2}$ & $9.410^{-11}$ & & & \\
\hline
\end{tabular}



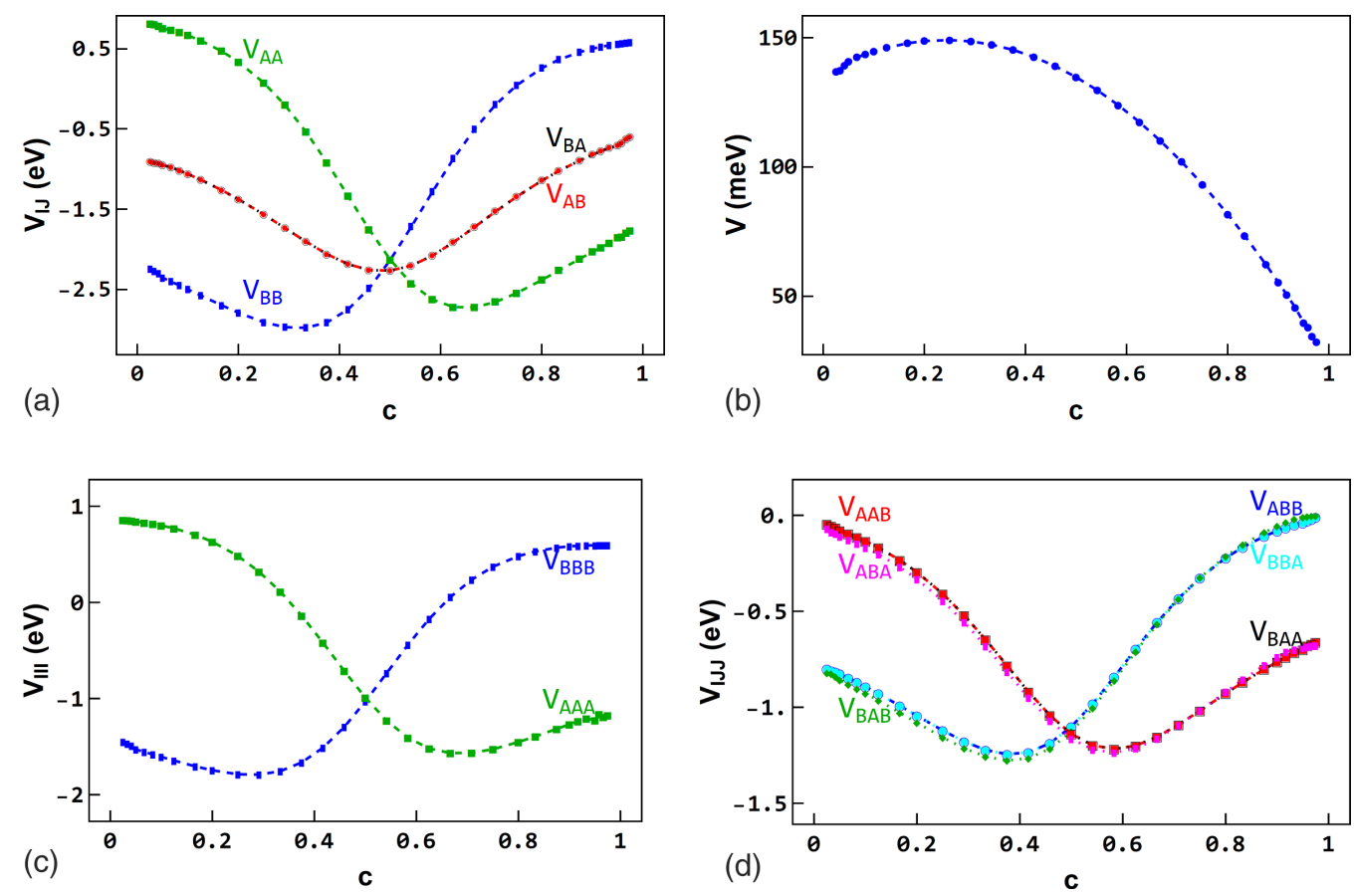

FIG. 4. Evolution as a function of the concentration $c$ of concentration-dependent effective cluster interactions (ECIs) issued from the cluster expansion (CE): (a) pairs, (b) effective alloy pair interaction $V=\left(V_{A A}+V_{B B}-V_{A B}-V_{B A}\right) / 2$, (c) and (d) triplets. (a) Green squares: $V_{A A}$, blue rectangles: $V_{B B}$, red points: $V_{A B}$, black circles: $V_{B A}$. (c) Green squares: $V_{A A A}$, blue rectangles: $V_{B B B}$. (d) Red squares: $V_{A A B}$, black empty squares: $V_{B A A}$, cyan points: $V_{B B A}$, blue circles: $V_{A B B}$, magenta rectangles: $V_{A B A}$, green diamonds: $V_{B A B}$.

some phantom interactions are introduced, i.e., interactions that are not in the model itself. The site energies depend on the concentration and on the first-neighboring atoms, while the $n$-tuple interactions do not depend on the concentration (for the concentration-independent CE case) and depend on the environment up to the $n$th neighboring shell. Compared with a standard fit where the range of ECIs is not related to an underlying Hamiltonian [58], fitting ECIs to site energies imposes a special treatment of excess variables to limit the range of ECIs to the range of the energetic model. Thus, we introduce into the tuple interactions the concentration dependency to reconstruct the site energies. If we take again the example of an atom $A$ surrounded on both sides by an atom $A$, two triplets involve second-neighboring atoms (see Fig. 5). These two triplets provide information that is not included in the initial energetic model. Interactions involving sites other than the nearest neighbors (the only sites considered in the site energies) are replaced by a mean-field development: $V_{I A A}=c V_{A A A}+(1-c) V_{B A A}$ and $V_{A A J}=c V_{A A A}+(1-c) V_{A A B}$. This development can be extended to the other triplets and to $n$-tuples.
The site energies can be written as a sum over the $n$-tuple site energies:

$$
E_{I}^{p}(n)=\sum_{j=2, \ldots, n} E V_{I}^{p}(j)
$$

with the $n$-tuple site energies in the form of

$$
E V_{I}^{p}(n)=\frac{1}{n} \sum_{k=1, \ldots, n} V_{n}^{k}\left(I_{k-1}, I_{k}, I_{k+1}\right) .
$$

Here, $V_{n}^{k}\left(I_{k-1}, I_{k}, I_{k+1}\right)$ represents the $n$-tuple with the central atom $I_{k}$ located at the $k$ th site of the $n$-chain. The chemical nature of the first-neighboring atoms of the atom $I_{k}, I_{k-1}$, and $I_{k+1}$ is given by the value of $p: I_{k-1}$ and $I_{k+1}$ are two atoms $B$ (respectively $A$ ) for $p=0$ (respectively $p=2$ ), one atom $A$ and one atom $B$ for $p=1$. The set of all possible $n$-tuples is defined by the value of $k$ which varies from 1, when the atom $I$ is located at the left end of the chain, to $n$, when it is at the right end. Each excess variable, i.e., each interaction involving sites beyond the first neighbors of $I$, is weighted by the probability to be occupied by an atom $A$ or $B$ :

$$
V_{n}^{k}\left(I_{k-1}, I_{k}, I_{k+1}\right)=\sum_{j=0}^{m_{k}} c^{j}(1-c)^{m_{k}-j}\left[\sum_{\sigma} \tilde{V}_{n}^{k, j}\left(I_{k-1}, I_{k}, I_{k+1}\right)\right] \text { with } \quad m_{k}=\max (n-k-1, k-2),
$$

with $\sigma$ the set of all possible $n$-tuples $\tilde{V}_{n}^{k, j}\left(I_{k-1}, I_{k}, I_{k+1}\right)$, with $I_{k}$ on the $k$ th site, $j$ atoms $A$, and $\left(m_{k}-j\right)$ atoms $B$ on the excess sites. For a better understanding, the cases of pairs and triplets are detailed in Appendix B. 


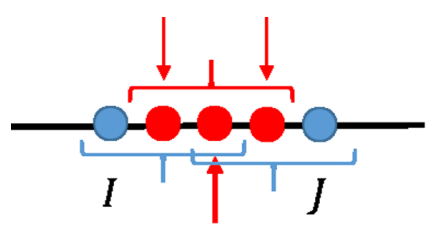

FIG. 5. Schematic representation of triplets involved for a central atom $A$ (arrow up) surrounded by two atoms $A$ (arrows down). Atoms $I$ and $J$ (blue) are unknown.

Here, $n$-tuple site energies $E V_{I}^{p}(n)$ are thus polynomial in $c$ of order $(n-2)$.

Recall now that the Hamiltonian of a RSS binary alloy can be written in the following form:

$$
H(c)=N_{a t} \sum_{p=0}^{Z} C_{Z}^{p} c^{p}(1-c)^{Z-p}\left[c E_{A}^{p}(c)+(1-c) E_{B}^{p}(c)\right] .
$$

Here, $C_{Z}^{p}$ is the number of ways that $p A$ neighbors can be chosen among $Z$ neighbors independently of their order.
Equation (9) enables us to predict the order of $H(c)$ from the order of site energies. Table II presents examples of the relationship between the highest terms of $H(c)$ as a function of the highest term of site energy.

For site energies that do not depend on $p$ and that are described by a polynomial of order $X$ in $c, H(c)$ is of order $X+1$. For site energies that are univariate polynomials in $p$, of order $Y, H(c)$ is also a univariate polynomial but in $c$ and of order $Y+1$. In other words, the dependency of site energies on local concentration (in $p$ ) is transformed into a dependency in $c$ of order $Y+1$. This general rule has some exceptions due to accidental cancellation of the highest term as a function of $Z$, as Table II shows, for $Y=3$, the order of $H(c)$ is 3 (and not 4 ) for $Z=2$. In general, when the highest term of the site energies is $c^{X} p^{Y}$, the Hamiltonian order in $c$ is $X+Y+1$.

On the other hand, since the $n$-tuples of site energies with constant ECIs are $c$ polynomials of order (n-2), EQS. (6)-(9) yield a Hamiltonian of order $(n-1)$, and thus, $n=X+Y+2$. For concentration-dependent ECIs, the mean-field approximation of a disordered solid solution (see Appendix $\mathrm{C}$ of Ref. [47]) gives the length of $n$-tuples: $n=Y+1$.
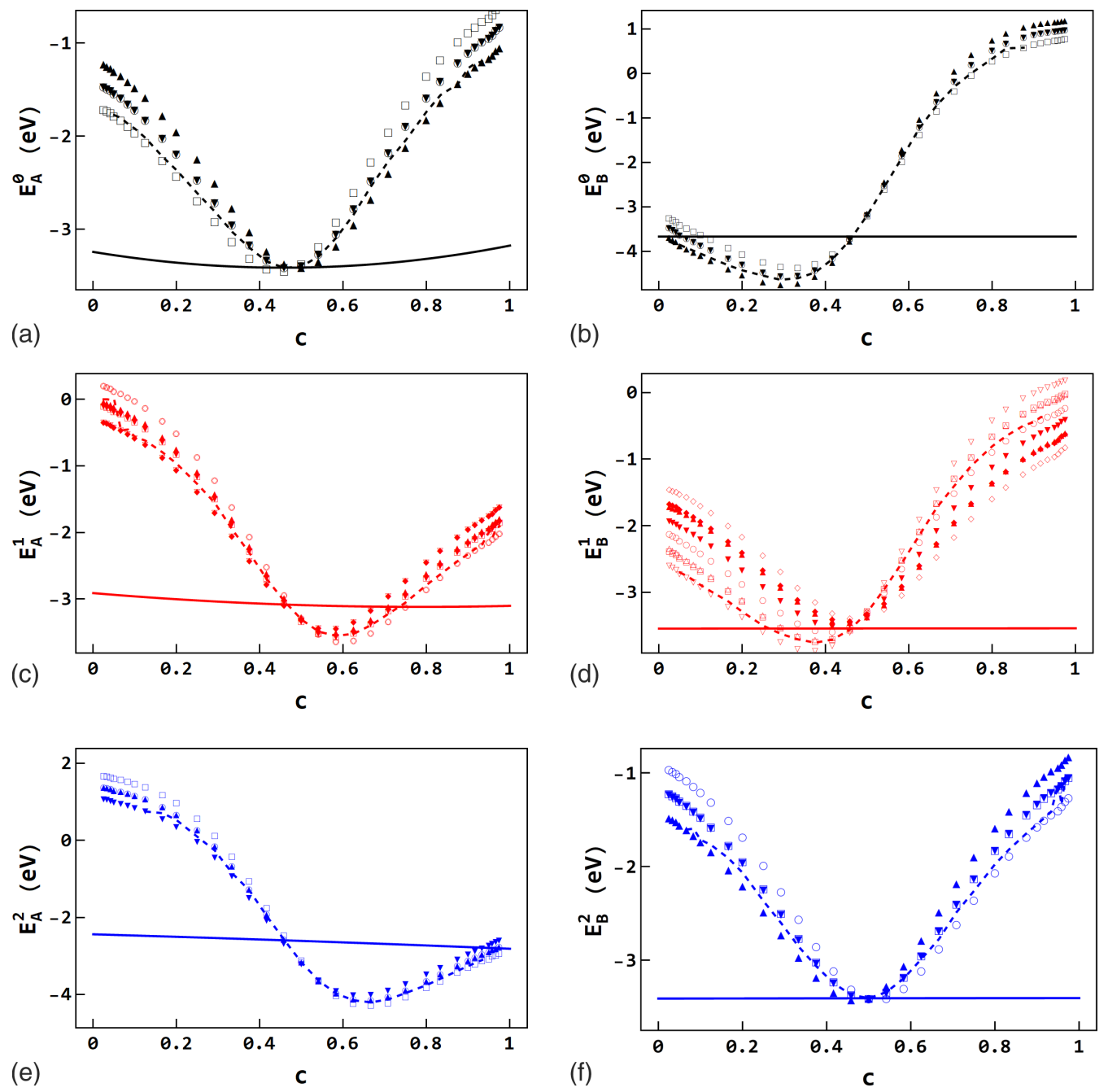

FIG. 6. Evolution as a function of the concentration $c$ of effective site energies (ESEs) (a)-(f) issued from the energetic model (continuous lines) and calculated with concentration-dependent effective cluster interactions (ECIs) issued from the cluster expansion (CE) from the average of all configurations (dashed lines) and with unknown sites occupied by $A$ or $B$ (symbols). 
TABLE II. Highest term of $H(c)$ [Eq. (9)] as a function of the highest term of the site energy $E_{I}^{p}(c)(I=A$ or $B)$.

\begin{tabular}{|c|c|c|c|c|c|c|}
\hline$E_{I}^{p}(c)$ & cte & $c$ & $c^{2}$ & $p$ & $p^{2}$ & $p^{3}$ \\
\hline$E_{I}^{p}(c)$ & & & & & $p^{2} c^{2}$ & $p^{2} c^{3}$ \\
\hline
\end{tabular}

To summarize, when the highest term of the site energies is $c^{X} p^{Y}$, the Hamiltonian order is $X+Y+1$, and the length of $n$-tuples independent of concentration is $n=X+Y+2$, and $n=Y+1$ for concentration-dependent $n$-tuples.

For the chosen energetic model [Eq. (1)], $X=2$ and $Y=2$, we therefore expect to reproduce site energies with concentration-independent ECIs extending up to sextuples $(X+Y+2)$, and triplets $(Y+1)$ for concentrationdependent interactions.

\section{B. ECIs fitting from site energies}

\section{Concentration-independent ECIS}

Pair, triplet, quadruplet, etc. interactions were determined by a least square fit from the site energies given by Eq. (1) for values of $c$ between 0 and 1 with a step of 0.02 . The range of $n$-tuples is gradually enlarged until a satisfactory agreement is reached. Figure 7 shows the resulting site energies by considering all $n$-tuples up to 6-tuples as expected. Note that the order of the 6-tuple interactions $V_{6}^{k}\left(I_{-1}, I, I_{1}\right)$ in $c$ being 4 , and those of site energies being 2 , the highest coefficients must be nil to have agreement. Numerically, these terms are very small $\sim 10^{-12}$.

The RMSE decreases with the $n$-tuple increase (Fig. 8, Table I). We now use these interactions to predict the total energy of structures. The number of structures being larger than the number of site energy values used for fitting the ECIs, the RMSE is lower than for the site energies (Fig. 8, Table I). We thus show that sextuple interactions enable us to fit both the site energies and the total energy of configurations.

\section{Concentration-dependent ECIs}

We determine then the weighted concentration-dependent ECIs from values of the site energies. As predicted by the Hamiltonian order analysis, pairs and triplets are sufficient to reproduce the site energies in the whole range of concentration. The interactions provide a perfect estimation of the total energy of the different configurations for both random and ordered configurations (see Table I). Remember that the definition of site energies [Eq. (2)] assumes that the quadratic term is an additional energy to the linear term. The linear term corresponds to the pair interactions, and the quadratic terms can be considered as the contribution of triplets. To be consistent with this definition, it is necessary to adjust first the pairs and then the triplets. Hence, the site energies are well fitted with the pairs for $p=0$ and $Z$. For the other values of $p$ (only $p=1$ for the $1 \mathrm{D}$ case), triplets are necessary to reproduce the curvature of the site energies. In this way, triplets reflect an excess energy as compared with the pair interactions. Of course, a simultaneous adjustment would have given other ECIs, we know that the adjustment procedure has a definite effect on $\mathrm{CE}$ [41]. Let us emphasize that, for any given concentration, the four unknown pair interactions are determined from four site energies ( $E_{I}^{p}$ with $p=0$ and $Z$ ), so the pair interactions have only one solution. The number of triplets to be estimated is 8. The six equations (see Appendix B) show that $V_{A A A}, V_{B B B}$, $V_{A B A}$, and $V_{B A B}$ can be determined without ambiguity. The sums $\left(V_{A A B}+V_{B A A}\right)$ and $\left(V_{A B B}+V_{B B A}\right)$ can also be identified, but not the triplets individually. Therefore, the triplets are only locally identifiable from the site energies whatever the value of concentration considered [59-61].

The evolution of pair and triplet interactions with concentration (Fig. 9) is very different from that obtained with concentration-dependent ECIs from the structures (Fig. 4). This result is not surprising because the ECIs are determined from site energies rather than configuration energies, and all excess variables are weighted by the mean-field approximation. The mixed pair interactions are not symmetrical [Fig. 9(a)], in accordance with the definition of site energies. The pair interactions being adjusted to the extreme values of
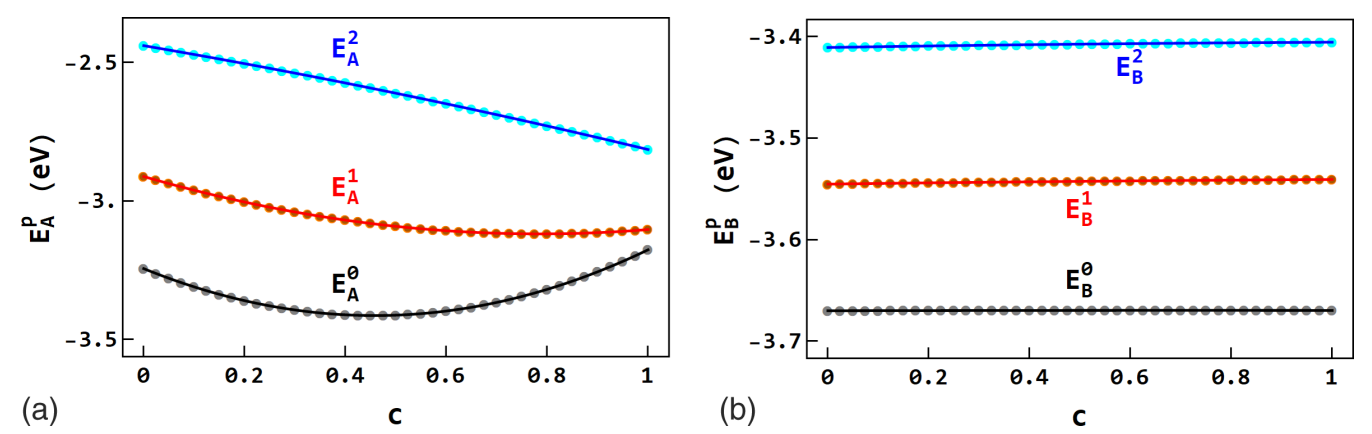

FIG. 7. Evolution of the effective site energies (ESEs) of an atom (a) $A$ and (b) $B$ as a function of $c$. Comparison between the fit by concentration-independent effective cluster interactions (ECIs) until sextuples (points) and the energetic model (continuous lines). The number $p$ of $A$ neighbors is equal to 0 (black), 1 (red), and 2 (blue). 


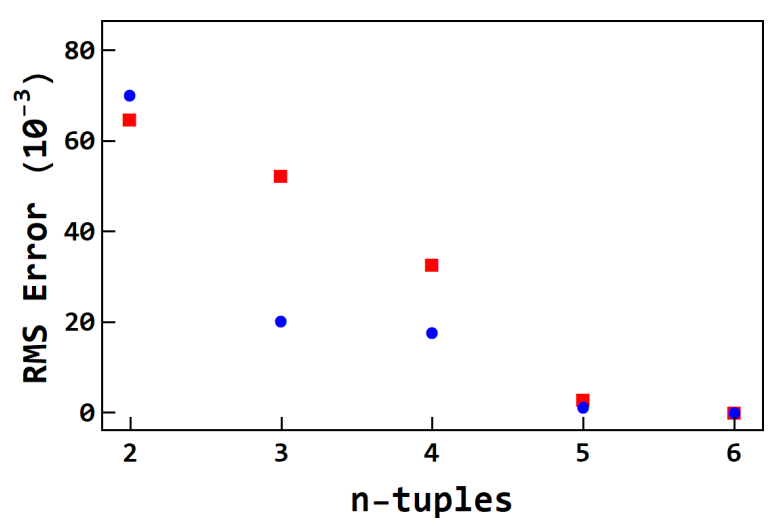

FIG. 8. Evolution of the root-mean-square error of the site energies (red squares) and of the energy of structures (blue points) as a function of the length of $n$-tuples. The concentration-independent $n$-tuples are fitted from the site energies.

the site energies (i.e., for $p=0$ and $Z$ ) and the slopes for $A$ and $B$ corresponding, respectively, to $V_{A A}-V_{A B}$ and $V_{B A}-V_{B B}$, there is no reason for $V_{A B}$ to be equal to $V_{B A}$. Two symmetrical relationships $V_{A B B}=V_{B B A}$ and $V_{A A B}=V_{B A A}$ are observed for the triplets [Fig. 9(c)], but other sets of values could have been obtained leading to the same sums $\left(V_{A A B}+V_{B A A}\right)$ and $\left(V_{A B B}+V_{B B A}\right)$.

Figure 10(a) shows that the alloy pair interaction $V_{p}(c)=\left\{V_{A A}(c)+V_{B B}(c)-\left[V_{A B}(c)+V_{B A}(c)\right]\right\} / 2$ is in perfect agreement with that given by the site energy model $\left[\left(E_{A}^{Z}-E_{A}^{0}\right)-\left(E_{B}^{Z}-E_{B}^{0}\right)\right] / 2$. Here, $V_{p}$ is positive throughout the concentration range. That means that the pair interactions tend to form heteroatomic bonds. The curvatures of the site energies of $A$ and $B$ atoms are also well reproduced by the triplet site energy $E V_{A}^{1}(n=3)$ [Fig. 10(b)] and $E V_{B}^{1}(n=3)$ [Fig. 10(c)].

The successive optimization of $n$-tuple interactions from site energies allows one to take advantage of the formalism developed to express the thermodynamic driving forces of the $1 \mathrm{D}$ alloy via ECIs.

\section{Discussion}

The adjustment of the $n$-tuple interactions on the local energies shows the presence of additional variables that are not included in the initial energetic model. This raises the question of the physical meaning of the parameters obtained in the $\mathrm{CE}$ on the structure energies. Different sets of interactions, with different ranges, may be obtained in classic $\mathrm{CE}$ [58]. It is therefore not possible that all sets are compatible with the range of the original model (which is usually unknown). CE with concentration-independent ECIs leads to $n$-tuples that are considerably more extended than the original energetic model since site energies are restricted to the nearest neighbors. It means that $\mathrm{CE}$ adds artificial dependencies that do not exist. These ECIs are coefficients useful to describe the system but which do not have the physical meaning of interactions. This formal study, which is based on a well-defined model, shows that CE can lead to misleading meanings. We believe this is a recurring problem because there is always the risk of introducing excess variables into the $n$-tuples that are not in the original energetic model. In the case of concentration-dependent ECIs, the dataset of energies is perfectly reproduced with pairs and triplets. Some triplets involve second neighbors that are not in the initial model.
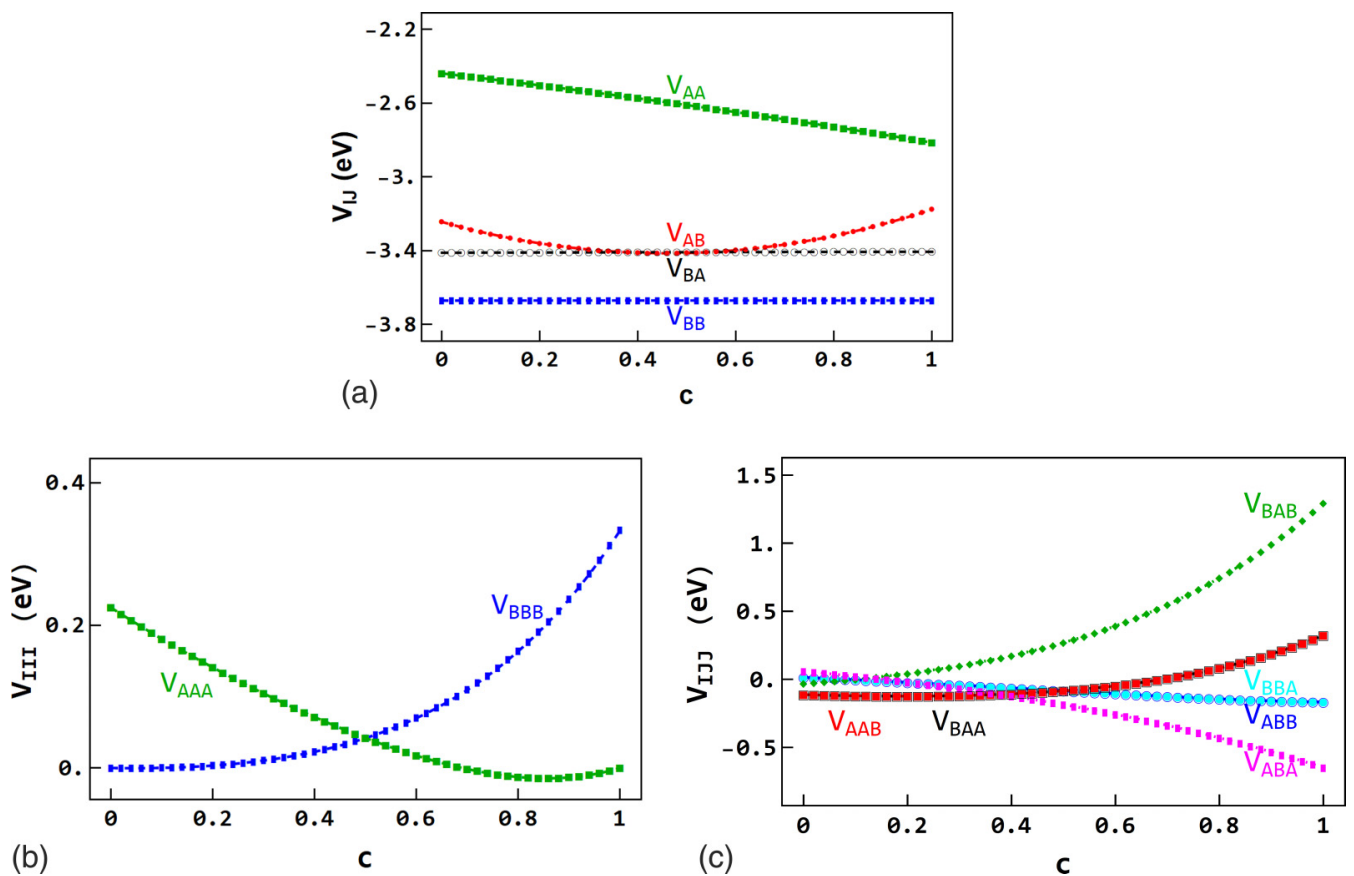

FIG. 9. Evolution as a function of the concentration $c$ of concentration-dependent effective cluster interactions (ECIs) issued from the site energies: (a) pairs, (b) and (c) triplets. (a) Green squares: $V_{A A}$, blue rectangles: $V_{B B}$, red points: $V_{A B}$, black circles: $V_{B A}$. (b) Green squares: $V_{A A A}$, blue rectangles: $V_{B B B}$. (c) Red squares: $V_{A A B}$, black empty squares: $V_{B A A}$, cyan points: $V_{B B A}$, blue circles: $V_{A B B}$, magenta rectangles: $V_{A B A}$, green diamonds: $V_{B A B}$. 


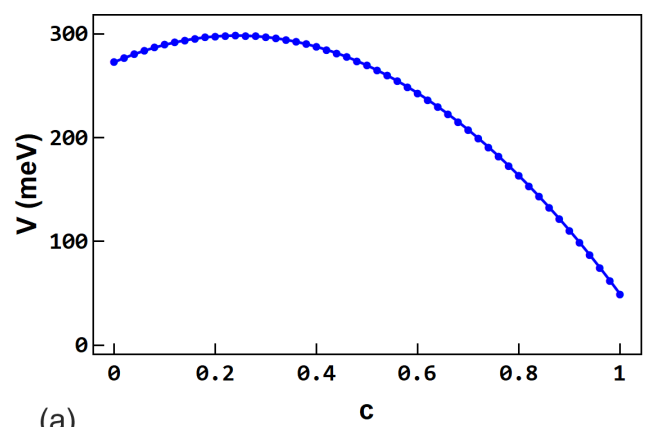

(a)
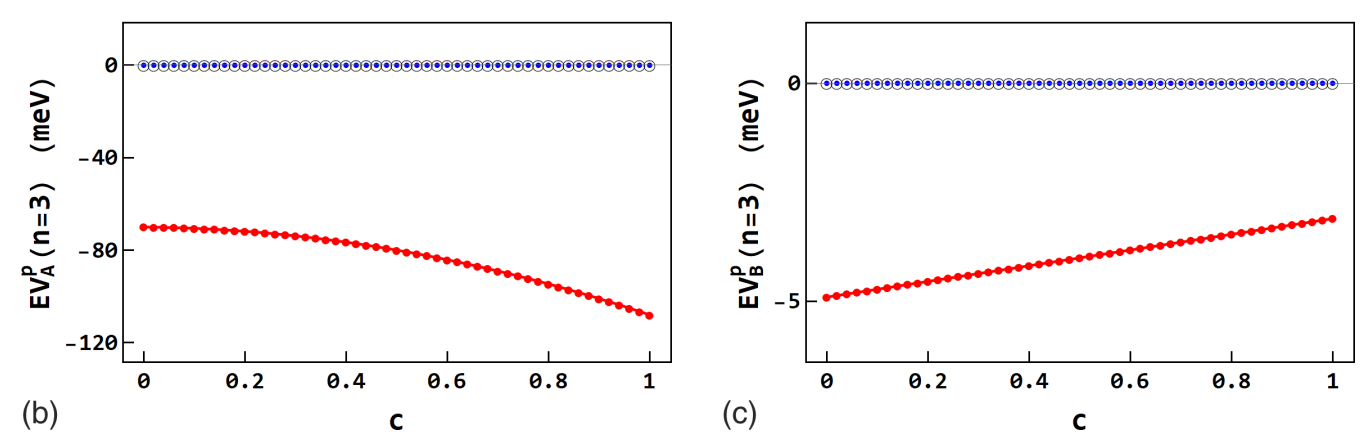

FIG. 10. (a) Effective alloy pair interaction $V=\left(V_{A A}+V_{B B}-V_{A B}-V_{B A}\right) / 2$ and (b) curvatures of $A$ and (c) $B$ site energies calculated from concentration-dependent effective cluster interactions (ECIs) issued from the site energies (points) and the energetic model (continuous lines). (b) and (c) The number $p$ of $A$ neighbors is equal to 0 (black), 1 (red), and 2 (blue).

The fact that CE does not reproduce local energies while it reproduces global energies very well may be problematic for the study of point defects. Fitting tuple interactions to local energies should instead make sense.

\section{THERMODYNAMIC DRIVING FORCES OF ALLOYS}

The ESEs allow one to decompose the mixing enthalpy and permutation enthalpy into chemical and elastic contributions. This analysis provides insight into the interplay between competing SRO and long-range order interactions, which give rise to complex phase diagrams. In this section, we express this decomposition in terms of pair and triplet interactions that have been optimized to reproduce site energies.

The Hamiltonian contains pair and triplet contributions:

$$
H(c)=H_{\text {pairs }}(c)+H_{\text {triplets }}(c),
$$

with

$$
\begin{aligned}
H_{\mathrm{pairs}}(c) / N_{a t}= & \frac{Z}{2} V_{B B}(c)+\frac{Z}{2}\left[V_{A A}(c)-V_{B B}(c)\right] c \\
& -Z c(1-c) V_{p}(c),
\end{aligned}
$$

and

$$
\begin{aligned}
H_{\text {triplets }}(c) / N_{a t}= & Z(Z-1) c(1-c)\left[c E V_{A}^{1}(n=3)\right. \\
& \left.+(1-c) E V_{B}^{1}(n=3)\right] .
\end{aligned}
$$

Triplet site energies $E V_{A}^{1}(n=3)$ and $E V_{B}^{1}(n=3)$ are detailed in Appendix B.

The mixing enthalpy corresponds to the formation energy of a RSS:

$$
\Delta H_{\mathrm{RSS}}^{\mathrm{mix}}(c)=H(c) / N_{a t}-\left[c E_{\mathrm{Coh}}^{A}+(1-c) E_{\mathrm{Coh}}^{B}\right]
$$

with $\quad E_{\mathrm{Coh}}^{A}=E_{A}^{Z}(c=1)=\frac{Z}{2} V_{A A}(c=1) \quad$ and $\quad E_{\mathrm{Coh}}^{B}=$ $E_{B}^{0}(c=0)=\frac{Z}{2} V_{B B}(c=0)$, which is then written as the sum of the chemical $\Delta H_{\mathrm{Chem}}^{\mathrm{mix}}(c)$ and elastic or size contributions $\Delta H_{\text {Size }}^{\operatorname{mix}}(c)$ :

$$
\Delta H_{\mathrm{RSS}}^{\mathrm{mix}}(c)=\Delta H_{\mathrm{Chem}}^{\mathrm{mix}}(c)+\Delta H_{\mathrm{Size}}^{\mathrm{mix}}(c),
$$

where the chemical contribution depends on the pair and the triplet contributions

$$
\Delta H_{\text {Chem }}^{\text {mix }}(c)=\Delta H_{\text {pairs }}^{\text {mix }}(c)+\Delta H_{\text {triplets }}^{\text {mix }}(c) .
$$

The different terms have the following expressions:

$$
\begin{aligned}
\Delta H_{\text {pairs }}^{\operatorname{mix}}(c)= & -Z c(1-c) V_{p}(c), \\
\Delta H_{\text {triplets }}^{\operatorname{mix}}(c)= & Z(Z-1) c(1-c)\left[c E V_{A}^{1}(n=3)\right. \\
& \left.+(1-c) E V_{B}^{1}(n=3)\right], \\
\Delta H_{\text {Size }}^{\operatorname{mix}}(c)= & \frac{Z}{2}\left\{c\left[V_{A A}(c)-V_{A A}(1)\right]\right. \\
& \left.+(1-c)\left[V_{B B}(c)-V_{B B}(0)\right]\right\} .
\end{aligned}
$$

In the size contribution, $V_{A A}(c)-V_{A A}(c=1)$ and $V_{B B}(c)-$ $V_{B B}(c=0)$ correspond to the variation of $A A$ and $B B$ interactions with the concentration relative to respective pure constituent. This dependence on concentration comes essentially from elastic effect.

The mixing enthalpy and its contributions can be seen in Fig. 11(a). The mixing enthalpies directly calculated for each configuration are dispersed around the mean field mixing enthalpy values [Eqs. (14)-(16)]. Here, $\Delta H_{\mathrm{RSS}}^{\operatorname{mix}}(c)$ is negative for $c \leqslant 0.8$ and slightly positive for $c \geqslant 0.8$. Elastic and chemical effects are in competition. The chemical contribution tends to form heteroatomic bonds since the chemical contribution is 

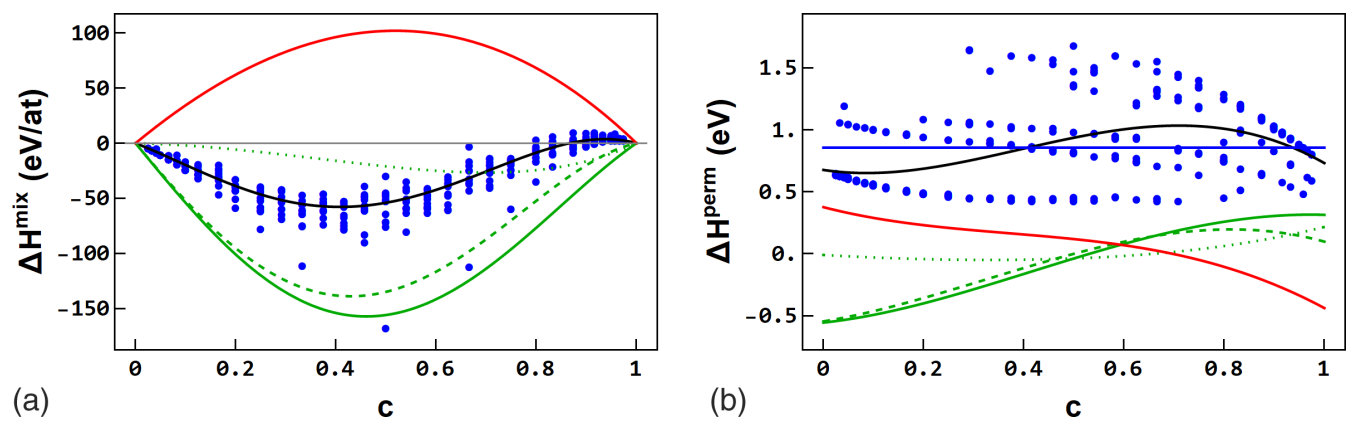

FIG. 11. (a) $\Delta H^{\text {mix }}$ and (b) $\Delta H^{\text {perm }}$ and evolution as a function of the concentration $c$ (in black) and decomposition of the different contributions: $\Delta H_{\mathrm{Coh}}^{\mathrm{perm}}$ (in blue), $\Delta H_{\text {Size }}^{\text {perm } / \mathrm{mix}}$ (in red), $\Delta H_{\text {Chem }}^{\text {perm } / \text { mix }}$ (in green), $\Delta H_{\text {Pairs }}^{\text {perm } / \text { mix }}$ (dashed green), $\Delta H_{\text {Triplets }}^{\text {perm } / \text { mix }}$ (dotted green) with $\Delta H_{\text {Chem }}^{\text {permix }}=\Delta H_{\text {Pairs }}^{\text {perm }}+\Delta H_{\text {Triplets }}^{\text {perm }}$. Blue points are the results of direct calculations for all configurations with the concentrationdependent pairs and triplets issued from the fit of the site energies.

negative. Let us detail the chemistry effect. The contribution of the triplets is slightly negative, almost nil, whatever the concentration. That of pairs is much more important. The SRO parameter is therefore mainly driven by the pairs. On the contrary, the size contribution is positive over the whole range of concentration. That means that the size effect favors the demixtion. While at low concentration the main contribution is chemistry, the size effect becomes predominant for $c \gtrsim 0.8$. At the highest concentrations, the size contribution leads to a long-range order to phase separation.

The ESE model also provides the analysis of the permutation enthalpy $\Delta H_{\mathrm{RSS}}^{\mathrm{perm}}$ (the enthalpy change when turning an atom $B$ to $A$ ), which is the key quantity that controls the composition of equilibrium configurations. The enthalpy of permutation is derived from either the Hamiltonian or the mixing enthalpy. It can be expressed as a function of the ECIs as well as the contributions according to the three effects rule in cohesive $\Delta H_{\text {Coh }}^{\text {perm }}$, chemical $\Delta H_{\text {Chem }}^{\text {perm }}$, and size $\Delta H_{\text {Size }}^{\text {perm }}$ effects:

$$
\Delta H_{\mathrm{RSS}}^{\mathrm{perm}}(c)=\Delta H_{\mathrm{Coh}}^{\mathrm{perm}}(c)+\Delta H_{\mathrm{Chem}}^{\mathrm{perm}}(c)+\Delta H_{\text {Size }}^{\mathrm{perm}}(c) .
$$

For the sake of brevity, all details are given in Appendix C.

The mixing enthalpy and the permutation enthalpy provide the same information but in different ways. Whereas the sign of the mixing enthalpy indicates that the alloy tends to phase separation $\left(\Delta H_{\mathrm{RSS}}^{\mathrm{mix}}>0\right)$ or to form ordered structures $\left(\Delta H_{\mathrm{RSS}}^{\text {mix }}<0\right)$, the sign of the slope of the permutation enthalpy gives the tendency of the alloys since the permutation enthalpy is the derivative of the mixing enthalpy [see Eq. (B1)]. A positive (respectively negative) slope characterizes a tendency to form ordered structures (respectively to phase separation).

The enthalpy of permutation and the cohesive, chemical, and elastic contributions are shown in Fig. 11(b). The permutation enthalpy is also determined for each configuration. It corresponds to the change in energy when a random $B$ atom of a given configuration is replaced by an $A$ atom. The direct values are consistent with $\Delta H_{\mathrm{RSS}}^{\mathrm{perm}}(c)$ but quite scattered. Here, $\Delta H_{\mathrm{RSS}}^{\text {perm }}(c)$ has a nonmonotonic behavior. For $c<0.8$, when $\Delta H_{\mathrm{RSS}}^{\mathrm{mix}}$ is negative, the slope of $\Delta H_{\mathrm{RSS}}^{\mathrm{perm}}(c)$ is positive; it becomes negative for $c>0.8$, when $\Delta H_{\mathrm{RSS}}^{\mathrm{mix}}$ is positive. The slope of the triplet contribution is close to 0 ; the slope of the chemical contribution is thus given by the slope of the pairs, and it is positive. Conversely, the slope of $\Delta H_{\text {Size }}^{\text {perm }}$ is negative.
At high concentration, the variation of $\Delta H_{\text {Size }}^{\text {perm }}$ with $c$ is the leading contribution.

The same decomposition is obtained from $\mathrm{CE}$ on structure energies with concentration-dependent ECIs. The formulas are given in Appendix D. CE that depends on $c$ gives access to the decomposition.

Determining the ECIs from site energies thus provides a clear distinction between the chemical and elastic driving forces that control the phase diagrams. This is a great advantage compared with Calphad, which consists of writing the mixing enthalpy as a polynomial function and determining the coefficients from thermodynamic, experimental, or $a b$ initio data $[49,50]$.

\section{DISCUSSION}

One may wonder if the method developed here for a simplified case can be extended to more complex systems such as 2D or three-dimensional (3D) systems, semi-infinite systems, defects, multicomponent alloys, or others.

The main difference between 2D and 3D systems and the 1D system is related to the choice of the clusters in CE to get an optimal truncation. Rules have been established to unambiguously define a set of clusters [41]. Thus, when a certain cluster is included in CE, then all subclusters (in range and numbers of atoms) must be included. The formalism is successfully applied to $2 \mathrm{D}$ systems for square $(Z=4)$ and hexagonal $(Z=6)$ structures. We believe that the formalism can therefore be developed for 3D systems.

The extension of this approach to inhomogeneous systems, such as surfaces or defects, is more delicate. The reference state chosen for a homogeneous system is the RSS. For inhomogeneous systems, the reference state must be chosen carefully. For example, for the study of a surface alloy on a pure $B$ substrate, a completely random reference state is too far from the equilibrium state. In this example, it is necessary to consider a RSS in the surface plane on a pure substrate in $B$ and to determine the site energies of the atoms $A$ and $B$ of the surface plane as a function of the local composition and the global composition (in the surface plane), as well as the site energies of the $B$ atoms of the subsurface plane first neighbors of the surface plane. The formalism, extended to the surface plane and to the $B$-plane first neighbors of the surface plane, allows us to correctly describe the thermodynamics. 
More generally, the extension to inhomogeneous systems is possible, provided that a reference state close to the equilibrium state is chosen, but not to the high energy states as well as CE [41].

This formalism can also be generalized to the case of pseudobinaries such as mixed oxides $A_{x} B_{1-x} \mathrm{O}_{2}$. In this case, the reference state is a RSS on the cationic lattice, and the energies of atoms $A, B$, and $\mathrm{O}$ are functions of local (the local composition in atoms $A$ ) and global (the nominal composition) variables. Study of $\mathrm{Sn}_{x} \mathrm{Ti}_{1-x} \mathrm{O}_{2}$ is currently under progress.

\section{CONCLUSIONS}

To summarize, we have successively implemented CE based on the total energy of configurations and on the site energies of RSSs. We used an energetic model limited to the first neighbors, and we considered a 1D system to restrict the discussion to the range of ECIs without considering the shape of the clusters.

First, we show that configurational CE, with fairly longrange ECIs (up to sextuples), gives a poor prediction of the mixing enthalpy of ordered and RSSs configurations. The convergence of CE is significantly improved by considering the dependence of ECIs on concentration. These results are consistent with the literature. The mixing enthalpy is very well predicted with concentration-dependent pairs and triplets. Moreover, we show that concentration-dependent CE gives us access to the decomposition of driving forces.

Concentration-dependent ECIs fit the energies of the configurations but do not reproduce the initial energetic model. $\mathrm{By}$ testing the prediction of site energies, we show that $\mathrm{CE}$ leads to a larger range of $n$-tuples than the range of the initial energetic model; it therefore describes variables that are not considered. By using an analytical model, we highlight a problem that we believe to be quite frequent. One may think that the range of the $n$-tuples is often greater than the real interactions. In this case, we can no longer really talk about ECIs because the coefficients determined do not really have a physical meaning; they simply fit the energies of the structures.

By developing CE from site energies, the ECIs allow one to reproduce the energetic model and the energies of ordered and random structures. The site energy formalism provides simple and strict control of the terms necessary for the convergence of $\mathrm{CE}$. It also gives the dependence in concentration of all the $n$-tuples. This leads to an unambiguous distinction between the chemical or elastic origin of the thermodynamic forces involved in the phase diagrams. This represents a triple advantage compared with CE, at least if the energies per site can be obtained. This point remains an open question for $a b$ initio calculations. Even if it is not the case, the formalism of site energies proves to be very promising for a systematic analysis of the thermodynamics of bimetallic alloys and their defects (dislocations, surface, grain boundaries).

\section{ACKNOWLEDGMENTS}

Q. Lullien was supported by the French National Research Agency under the program CHARMMMAT ANR-11-LABX0039-grant. The authors would like to thank F. Soisson for very fruitful discussions.

\section{APPENDIX A: DRIVING FORCES FROM SITE ENERGIES}

We recall in this Appendix how the dependence of the site energies on local and global variables (i.e., the local composition $p$ and on the nominal concentration $c$ ) allows us to extract elastic and chemical effects. We first explain the calculation of the permutation enthalpy from the Hamiltonian of site energies [Eq. (9)].

The introduction of the site energies [Eq. (2)] in Eq. (9) leads to the following expression of the Hamiltonian:

$$
\begin{aligned}
\frac{\langle H\rangle}{N}= & E_{B}^{0}(c)+\left[E_{A}^{Z}(c)-E_{B}^{0}(c)\right] c-c(1-c)\left\{\left[E_{A}^{Z}(c)-E_{A}^{0}(c)\right]-\left[E_{B}^{Z}(c)-E_{B}^{0}(c)\right]\right\} \\
& +Z(Z-1) c(1-c)\left[c \varkappa_{A}(c)+(1-c) \varkappa_{B}(c)\right] .
\end{aligned}
$$

The dependence of the site energies on the local composition $p$ is transformed in the Hamiltonian into a dependence on $c$. The dependence on the local composition is then less explicit. However, writing the enthalpy of permutation allows us to separate two components. To do this, we first introduce the cohesive contribution $\left\{\Delta H_{\mathrm{Coh}}^{\mathrm{perm}}=\left(E_{\mathrm{Coh}}^{A}-E_{\mathrm{Coh}}^{B}\right)=\left[E_{A}^{Z}(1)-E_{B}^{0}(0)\right]\right\}$ :

$$
\Delta H^{\mathrm{perm}}=\Delta H_{\mathrm{Coh}}^{\mathrm{perm}}+\frac{1}{N} \frac{\partial\langle H\rangle}{\partial c}-\left[E_{A}^{Z}(1)-E_{B}^{0}(0)\right] .
$$

To transform the third term of the right-hand side, we add and subtract $\left[E_{A}^{Z}(c)-E_{B}^{0}(c)\right]$ :

$$
\Delta H^{\mathrm{perm}}=\Delta H_{\mathrm{Coh}}^{\mathrm{perm}}-\left[E_{A}^{Z}(c)-E_{B}^{0}(c)\right]+\frac{1}{N} \frac{\partial\langle H\rangle}{\partial c}+\left\{\left[E_{A}^{Z}(c)-E_{A}^{Z}(1)\right]-\left[E_{B}^{0}(c)-E_{B}^{0}(0)\right]\right\} .
$$

Then we decompose $\frac{\partial\langle H\rangle}{\partial c}$ into two derivatives $\frac{\partial\langle H\rangle}{\partial c}=\left.\frac{\partial\langle H\rangle}{\partial c}\right|_{a}+\left.\frac{\partial\langle H\rangle}{\partial c}\right|_{b}$, where $\left.\frac{\partial\langle H\rangle}{\partial c}\right|_{a}$ denotes that only the combinatorial sums of the site energies are differentiated (the site energies being fixed at a given value of $c$ ), whereas for $\left.\frac{\partial\langle H\rangle}{\partial c}\right|_{b}$, only the site energies are differentiated. Let us recall that the variation of the site energies with concentration is related to the elastic effect. Thus, the last term of the right-hand side, which is the difference in $c$ slope $\Delta_{c}\left(E_{A}^{Z}\right)-\Delta_{c}\left(E_{B}^{0}\right)$ at a given $p$ value $(Z$ for $A$ and 0 for $B)$, is also an elastic term. 
This decomposition allows us to define the chemical and elastic contributions:

$$
\Delta H_{\mathrm{Chem}}^{\mathrm{perm}}=-\left[E_{A}^{Z}(c)-E_{B}^{0}(c)\right]+\left.\frac{1}{N} \frac{\partial\langle H\rangle}{\partial c}\right|_{a}
$$

and

$$
\Delta H_{\text {Size }}^{\mathrm{perm}}=\left\{\left[E_{A}^{Z}(c)-E_{A}^{Z}(1)\right]-\left[E_{B}^{0}(c)-E_{B}^{0}(0)\right]\right\}+\left.\frac{1}{N} \frac{\partial\langle H\rangle}{\partial c}\right|_{b},
$$

which leads to

$$
\begin{aligned}
\Delta H_{\text {Chem }}^{\text {perm }}= & -(1-2 c)\left\{\left[E_{A}^{Z}(c)-E_{A}^{0}(c)\right]-\left[E_{B}^{Z}(c)-E_{B}^{0}(c)\right]\right\}+Z(Z-1)(1-2 c)\left[c \varkappa_{A}(c)+(1-c) \varkappa_{B}(c)\right] \\
& +Z(Z-1) c(1-c)\left[\varkappa_{A}(c)-\varkappa_{B}(c)\right] .
\end{aligned}
$$

We can see that $\Delta H_{\text {Chem }}^{\mathrm{perm}}$ depends on the difference in $p-$ slope $\Delta_{p}\left(E_{A}^{p}\right)-\Delta_{p}\left(E_{B}^{p}\right)$, with $\Delta_{p}\left(E_{I}^{p}\right)=E_{I}^{Z}-E_{I}^{0}$ at a given $c$ value. We have previously shown that terms which depend on the curvatures are due to the change for neighbors when a $B$ atom is turned into an $A$ atom [46].

The size contribution is written as

$$
\begin{aligned}
\Delta H_{\text {Size }}^{\text {perm }}= & \left\{\left[E_{A}^{Z}(c)-E_{A}^{Z}(1)\right]-\left[E_{B}^{0}(c)-E_{B}^{0}(0)\right]\right\}+\dot{E}_{B}^{0}(c)+\left[\dot{E}_{A}^{Z}(c)-\dot{E}_{B}^{0}(c)\right] c \\
& -c(1-c)\left\{\left[\dot{E}_{A}^{Z}(c)-\dot{E}_{A}^{0}(c)\right]-\left[\dot{E}_{B}^{Z}(c)-\dot{E}_{B}^{0}(c)\right]\right\} \\
& +Z(Z-1) c(1-c)\left[c \dot{\varkappa}_{A}(c)+(1-c) \dot{\varkappa}_{B}(c)\right],
\end{aligned}
$$

with $\dot{E}_{I}^{p}(c)=\partial E_{I}^{p}(c) / \partial c$.

The mixing enthalpy is then deduced via the following equation:

$$
\Delta H^{\mathrm{mix}}=\int_{0}^{c} \Delta H^{\mathrm{perm}}(u) d u-c \int_{0}^{1} \Delta H^{\mathrm{perm}}(u) d u,
$$

or more easily from its definition $\Delta H^{\mathrm{mix}}=\langle H\rangle / N-\left[c E_{\mathrm{Coh}}^{A}+(1-) E_{\mathrm{Coh}}^{B}\right]$ :

$$
\begin{aligned}
\Delta H^{\mathrm{mix}}= & -c(1-c)\left\{\left[E_{A}^{Z}(c)-E_{A}^{0}(c)\right]-\left[E_{B}^{Z}(c)-E_{B}^{0}(c)\right]\right\}+Z(Z-1) c(1-c)\left[c \varkappa_{A}(c)+(1-c) \varkappa_{B}(c)\right] \\
& +\left\{c\left[E_{A}^{Z}(c)-E_{A}^{Z}(1)\right]+(1-c)\left[E_{B}^{0}(c)-E_{B}^{0}(0)\right]\right\} .
\end{aligned}
$$

We recognize in the first term of the right-hand side the difference in $p-$ slope $\Delta_{p}\left(E_{A}^{p}\right)-\Delta_{p}\left(E_{B}^{p}\right)$ and, in the second term, the curvatures at a given $c$ value. These two terms are related to the local composition:

$$
\Delta H_{\text {Chem }}^{\mathrm{perm}}=-c(1-c)\left\{\left[E_{A}^{Z}(c)-E_{A}^{0}(c)\right]-\left[E_{B}^{Z}(c)-E_{B}^{0}(c)\right]\right\}+(Z-1) c(1-c)\left[c \varkappa_{A}(c)+(1-c) \varkappa_{B}(c)\right] .
$$

The third term of the right-hand side [Eq. (A9)] shows the variations of the site energies with the concentration at a fixed value of $p$; it is thus an elastic term:

$$
\Delta H_{\text {Size }}^{\text {perm }}=c\left[E_{A}^{Z}(c)-E_{A}^{Z}(1)\right]+(1-c)\left[E_{B}^{0}(c)-E_{B}^{0}(0)\right] .
$$

\section{APPENDIX B: PAIR AND TRIPLET SITE ENERGIES}

We note hereafter the site energies written according to the $n$-tuple interactions $E V_{I}^{p}(n)$ to distinguish them from the site energies defined as the initial energetic model. The pair interactions induce straightforwardly an equivalent expression of site energies:

$$
\begin{array}{lll}
E V_{A}^{0}(n=2)=V_{A B}, & E V_{A}^{1}(n=2)=\frac{1}{2}\left(V_{A A}+V_{A B}\right), & E V_{A}^{2}(n=2)=V_{A A} \\
E V_{B}^{0}(n=2)=V_{B B}, & E V_{B}^{1}(n=2)=\frac{1}{2}\left(V_{B A}+V_{B B}\right), & E V_{B}^{2}(n=2)=V_{B A} .
\end{array}
$$

Larger tuples involve neighboring shells beyond the range of the energetic model. These interactions are weighted by the probability that unknown occupation numbers being 1 (i.e., occupied by an atom $A$ ) is $c$ or being 0 (respectively $B$ ) is $(1-c$ ). Thus, for triplets, we get the following expressions for an atom $A$ :

$$
\begin{aligned}
& E V_{A}^{0}(n=3)=\frac{1}{3}\left\{\left[c V_{A B A}+(1-c) V_{A B B}\right]+V_{B A B}+\left[c V_{A B A}+(1-c) V_{B B A}\right]\right\}, \\
& E V_{A}^{1}(n=3)=\frac{1}{3}\left\{\left[c V_{A B A}+(1-c) V_{A B B}\right]+V_{A A B}+\left[c V_{A A A}+(1-c) V_{B A A}\right]\right\},
\end{aligned}
$$

or

$$
\begin{aligned}
& E V_{A}^{1}(n=3)=\frac{1}{3}\left\{\left[c V_{A A A}+(1-c) V_{A A B}\right]+V_{B A A}+\left[c V_{A B A}+(1-c) V_{B B A}\right]\right\} \\
& E V_{A}^{2}(n=3)=\frac{1}{3}\left\{\left[c V_{A A A}+(1-c) V_{A A B}\right]+V_{A A A}+\left[c V_{A A A}+(1-c) V_{B A A}\right]\right\}
\end{aligned}
$$


For a $B$ atom,

$$
\begin{aligned}
& E V_{B}^{0}(n=3)=\frac{1}{3}\left\{\left[c V_{B B A}+(1-c) V_{B B B}\right]+V_{B B B}+\left[c V_{A B B}+(1-c) V_{B B B}\right]\right\}, \\
& E V_{B}^{1}(n=3)=\frac{1}{3}\left\{\left[c V_{B A A}+(1-c) V_{B A B}\right]+V_{B B A}+\left[c V_{A B B}+(1-c) V_{B B B}\right]\right\}
\end{aligned}
$$

or

$$
\begin{aligned}
& E V_{B}^{0}(n=3)=\frac{1}{3}\left\{\left[c V_{B B A}+(1-c) V_{B B B}\right]+V_{A B B}+\left[c V_{A A B}+(1-c) V_{B A B}\right]\right\}, \\
& E V_{B}^{2}(n=3)=\frac{1}{3}\left\{\left[c V_{B A A}+(1-c) V_{B A B}\right]+V_{A B A}+\left[c V_{A A B}+(1-c) V_{B A B}\right]\right\}
\end{aligned}
$$

For $p=0$ or $Z$, the quadratic term is cancelled, and the site energies depend only on the linear term. Pair interactions therefore allow one to perfectly reproduce these site energies $E_{I}^{p}=E V_{I}^{p}(n=2)$ and $E V_{I}^{p}(n=3)=0$. Triplets are necessary to reproduce the site energies when $0<p<Z: E_{I}^{1}=E V_{I}^{1}(n=2)+E V_{I}^{1}(n=3)$. Triplets correspond to the excess energy compared with the pair interactions, so they fit the curvature term $E V_{I}^{1}(n=3)=\varkappa_{I}$.

\section{APPENDIX C: ENTHALPY OF PERMUTATION}

We detail in this Appendix the different contributions of the permutation enthalpy of a RSS written as a function of the weighted ECIs determined by CE from the site energies by applying the approach detailed in Appendix A. The permutation enthalpy is obtained by derivation of the enthalpy given by Eqs. (10)-(12):

$$
\Delta H^{\text {perm }}(c)=\Delta H_{\text {Coh }}^{\text {perm }}(c)+\frac{1}{N} \frac{\partial H(c)}{\partial c}-\frac{Z}{2}\left[V_{A A}(1)-V_{B B}(0)\right],
$$

where $\Delta H_{\mathrm{Coh}}^{\text {perm }}(c)=\frac{Z}{2}\left[V_{A A}(1)-V_{B B}(0)\right]$. Then we add and subtract $Z\left[V_{A A}(c)-V_{B B}(c)\right] / 2$, and we split the enthalpy of perturbation into three contributions:

$$
\Delta H^{\text {perm }}(c)=\Delta H_{\text {Coh }}^{\text {perm }}(c)+\Delta H_{\text {Chem }}^{\text {perm }}(c)+\Delta H_{\text {Size }}^{\text {perm }}(c),
$$

with

$$
\Delta H_{\mathrm{Chem}}^{\mathrm{perm}}=-\frac{Z}{2}\left[V_{A A}(c)-V_{B B}(c)\right]+\left.\frac{1}{N} \frac{\partial\langle H\rangle}{\partial c}\right|_{a}
$$

and

$$
\Delta H_{\text {Size }}^{\text {perm }}=\frac{Z}{2}\left\{\left[V_{A A}(c)-V_{A A}(1)\right]-\left[V_{B B}(c)-V_{B B}(0)\right]\right\}+\left.\frac{1}{N} \frac{\partial\langle H\rangle}{\partial c}\right|_{b}
$$

The elastic term is expressed as

$$
\begin{aligned}
\Delta H_{\text {Size }}^{\text {perm }}(c)= & \frac{Z}{2}\left\{\left[V_{A A}(c)-V_{A A}(1)\right]-\left[V_{B B}(c)-V_{B B}(1)\right]\right\}-Z c(1-c) \frac{\partial V(c)}{\partial c} \\
& +Z(Z-1) c(1-c)\left[c \frac{\partial E V_{A}^{1}(n=3)}{\partial c}+(1-c) \frac{\partial E V_{B}^{1}(n=3)}{\partial c}\right]+\frac{Z}{2}\left[c \frac{\partial V_{A A}(c)}{\partial c}+(1-c) \frac{\partial V_{B B}(c)}{\partial c}\right] .
\end{aligned}
$$

The chemical contribution is the sum of the pair and triplet contributions:

$$
\Delta H_{\text {Chem }}^{\text {perm }}(c)=\Delta H_{\text {pairs }}^{\text {perm }}(c)+\Delta H_{\text {triplets }}^{\text {perm }}(c),
$$

which are written as

$$
\begin{aligned}
\Delta H_{\text {pairs }}^{\text {perm }}(c) & =-Z(1-2 c) V_{p}(c), \\
\Delta H_{\text {triplets }}^{\text {perm }}(c) & =Z(Z-1)\left[c(2-3 c) E V_{A}^{1}(n=3)+(1-c)(1-3 c) E V_{B}^{1}(n=3)\right] .
\end{aligned}
$$

\section{APPENDIX D: DRIVING FORCES FROM CONCENTRATION-DEPENDENT ECIS}

It is also possible to separate the chemical and elastic contributions when the ECIs are determined directly from the structures, provided they are concentration dependent (with nonweighted ECIs).

In the mean-field approximation, the generalized Hamiltonian given by Eq. (5) is expressed as

$$
\langle H\rangle / N=\left\{Z\left[\tau(c)-V_{p}(c)\right] c+Z V_{p}(c) c^{2}+\frac{Z V_{B B}(c)}{2}\right\}+\frac{Z(Z-1)}{2}\left[R_{3}(c) c^{3}+R_{2}(c) c^{2}+R_{1}(c) c+V_{B B B}(c)\right],
$$


with

$$
\begin{aligned}
\tau(c) & =\frac{V_{A A}(c)-V_{B B}(c)}{2}, \quad V_{p}(c)=\frac{V_{A A}(c)+V_{B B}(c)-V_{A B}(c)-V_{B A}(c)}{2}, \\
R_{3}(c) & =V_{A A A}(c)-V_{B B B}(c)+\left[V_{A B B}(c)+V_{B A B}(c)+V_{B B A}(c)\right]-\left[V_{A A B}(c)+V_{A B A}(c)+V_{B A A}(c)\right], \\
R_{2}(c) & =\left[V_{A A B}(c)+V_{A B A}(c)+V_{B A A}(c)\right]+3 V_{B B B}(c)-2\left[V_{A B B}(c)+V_{B A B}(c)+V_{B B A}(c)\right], \\
R_{1}(c) & =\left[V_{A B B}(c)+V_{B A B}(c)+V_{B B A}(c)\right]-3 V_{B B B}(c) .
\end{aligned}
$$

The approach developed in Appendix A is applied to the generalized Hamiltonian [Eq. (D1)]. We first introduce the cohesive contribution $\left\{\Delta H_{\mathrm{Coh}}^{\text {perm }}=\left(E_{\mathrm{Coh}}^{A}-E_{\mathrm{Coh}}^{B}\right)=Z\left[V_{A A}(1)-V_{B B}(0)\right] / 2\right\}$ :

$$
\Delta H^{\text {perm }}=\Delta H_{\mathrm{Coh}}^{\text {perm }}+\frac{1}{N} \frac{\partial\langle H\rangle}{\partial c}-\frac{Z}{2}\left[V_{A A}(1)-V_{B B}(0)\right] .
$$

Then we add and subtract $\left[V_{A A}(c)-V_{B B}(c)\right] / 2$ :

$$
\Delta H^{\mathrm{perm}}=\Delta H_{\mathrm{Coh}}^{\mathrm{perm}}-\frac{Z}{2}\left[V_{A A}(c)-V_{B B}(c)\right]+\frac{1}{N} \frac{\partial\langle H\rangle}{\partial c}+\frac{Z}{2}\left\{\left[V_{A A}(c)-V_{A A}(1)\right]-\left[V_{B B}(c)-V_{B B}(0)\right]\right\},
$$

leading to

$$
\Delta H_{\mathrm{Chem}}^{\mathrm{perm}}=-\frac{Z}{2}\left[V_{A A}(c)-V_{B B}(c)\right]+\left.\frac{1}{N} \frac{\partial\langle H\rangle}{\partial c}\right|_{a}
$$

and

$$
\Delta H_{\text {Size }}^{\text {perm }}=\frac{Z}{2}\left\{\left[V_{A A}(c)-V_{A A}(1)\right]-\left[V_{B B}(c)-V_{B B}(0)\right]\right\}+\left.\frac{1}{N} \frac{\partial\langle H\rangle}{\partial c}\right|_{b}
$$

The final expressions are

$$
\Delta H_{\text {Chem }}^{\mathrm{perm}}=-Z(1-2 c) V_{p}(c)+\frac{Z(Z-1)}{2}\left[3 R_{3}(c) c^{2}+2 R_{2}(c) c+R_{1}(c)\right],
$$

and

$$
\begin{aligned}
\Delta H_{\text {Size }}^{\text {perm }}= & \frac{Z}{2}\left\{\left[V_{A A}(c)-V_{A A}(1)\right]-\left[V_{B B}(c)-V_{B B}(0)\right]\right\}+\left\{Z\left[\dot{\tau}(c)-\dot{V}_{p}(c)\right] c+Z \dot{V}_{p}(c) c^{2}+\frac{Z \dot{V}_{B B}(c)}{2}\right\} \\
& +\frac{Z(Z-1)}{2}\left[\dot{R}_{3}(c) c^{3}+\dot{R}_{2}(c) c^{2}+\dot{R}_{1}(c) c+\dot{V}_{B B B}(c)\right] .
\end{aligned}
$$

Then we write the mixing enthalpy and its three components.

The size contribution is expressed as the weighted sum of the variations of homoatomic interactions with the global concentration relative to the pure constituents:

$$
\begin{aligned}
\Delta H_{\text {Size }}^{\operatorname{mix}}(c)= & \frac{Z}{2}\left\{c\left[V_{A A}(c)-V_{A A}(1)\right]+(1-c)\left[V_{B B}(c)-V_{B B}(0)\right]\right\} \\
& +\frac{Z(Z-1)}{2}\left\{c\left[V_{A A A}(c)-V_{A A A}(1)\right]+(1-c)\left[V_{B B B}(c)-V_{B B B}(0)\right]\right\} .
\end{aligned}
$$

Thus, the chemical contribution of the mixing enthalpy is given by

$$
\Delta H_{\mathrm{Chem}}^{\operatorname{mix}}(c)=-c(1-c) Z V_{p}(c)-Z \frac{(Z-1)}{2} c(1-c)\left[R_{2}(c)+(1+c) R_{3}(c)\right] .
$$

Note that $\Delta H_{\text {Chem }}^{\operatorname{mix}}$ can also be written as

$$
\Delta H_{\text {Chem }}^{\text {mix }}(c)=-c(1-c) Z V(c)-c(1-c) Z \frac{(Z-1)}{2}(1-2 c) R_{3}(c),
$$

with $Z V=\sum_{n} Z_{n} V_{n}, Z_{n}$, and $V_{n}$ being, respectively, the coordination number and the effective pair interactions (EPIs) in the $n$th neighboring shell. The EPIs $V_{n}$ are obtained by considering the enthalpy difference $\Delta H_{n}$ of a RSS containing two isolated solute atoms and two solvent atoms in $n$ th-neighbor position (initial state) and two solute atoms in the $n$ th-neighbor position and two isolated solvent atoms (final state). Here, $V_{n}$ is related to $\Delta H_{n}$ by $\Delta H_{n}=(1-2 c) Z_{n} V_{n}$. This leads to

$$
V_{1}(c)=V_{p}(c)+(Z-1)\left(\frac{R_{2}(c)}{2}+\varkappa_{B}(c)+c R_{3}(c)\right)
$$


and

$$
V_{2}(c)=(Z-1)\left(-\varkappa_{B}(c)+c \frac{R_{3}(c)}{2}\right)
$$

with $\varkappa_{B}(c)=\left[V_{A B B}(c)+V_{B B A}(c)-V_{B B B}(c)-V_{A B A}(c)\right] / 2$; this term corresponds to the curvature of the site energies of $B$ atoms.

[1] J. M. Sanchez and D. de Fontaine, The fee Ising model in the cluster variation approximation, Phys. Rev. B 17, 2926 (1978).

[2] J. M. Sanchez and D. de Fontaine, Ordering in fcc lattices with first- and second-neighbor interactions, Phys. Rev. B 21, 216 (1980).

[3] J. M. Sanchez and D. de Fontaine, Ising model phase-diagram calculations in the fcc lattice with first- and second-neighbor interactions, Phys. Rev. B 25, 1759 (1982).

[4] R. Kikuchi, A theory of cooperative phenomena, Phys. Rev. 81, 988 (1951).

[5] G. Ceder, A derivation of the Ising model for the computation of phase diagrams, Comput. Mater. Sci. 1, 144 (1993).

[6] J. W. D. Connolly and A. R. Williams, Density-functional theory applied to phase transformations in transition-metal alloys, Phys. Rev. B 27, 5169 (1983).

[7] J. M. Sanchez, F. Ducastelle, and D. Gratias, Generalized cluster description of multicomponent systems, Physica A 128, 334 (1984).

[8] R. Biswas and D. R. Hamann, New classical models for silicon structural energies, Phys. Rev. B 36, 6434 (1987).

[9] F. H. Stillinger and T. A. Weber, Computer simulation of local order in condensed phases of silicon, Phys. Rev. B 31, 5262 (1985).

[10] R. Drautz, Atomic cluster expansion for accurate and transferable interatomic potentials, Phys. Rev. B 99, 014104 (2019).

[11] F. Ducastelle, Modules élastiques des métaux de transition, J. Phys. 31, 1055 (1970).

[12] V. Rosato, M. Guillopé, and B. Legrand, Thermodynamical and structural properties of f.c.c. transition metals using a simple tight-binding model, Philos. Mag. A 59, 321 (1989).

[13] F. Berthier, B. Legrand, and G. Tréglia, How to compare superficial and intergranular segregation? A new analysis within the mixed SMA-TBIM approach, Acta Mater. 47, 2705 (1999).

[14] F. Berthier, B. Legrand, and G. Tréglia, New structures and atomistic analysis of the polymorphism for the $\Sigma=5$ (210) [001] tilt boundary, Interface Sci. 8, 55 (2000).

[15] J. Creuze, F. Berthier, R. Tétot, and B. Legrand, Wetting and Structural Transition Induced by Segregation at Grain Boundaries: A Monte Carlo Study, Phys. Rev. Lett. 86, 5735 (2001).

[16] F. Berthier, J. Creuze, R. Tétot, and B. Legrand, Structural phase transition induced by interfacial segregation: a comparison between surface and grain boundary, Appl. Surf. Sci. 177, 243 (2001).

[17] R. Tétot, F. Berthier, J. Creuze, I. Meunier, G. Tréglia, and B. Legrand, $\mathrm{Cu}-\mathrm{Ag}$ (111) Polymorphism Induced by Segregation and Advacancies, Phys. Rev. Lett. 91, 176103 (2003).

[18] G. Tréglia, C. Goyhenex, C. Mottet, C. Legrand, and F. Ducastelle, Electronic structure of nanoalloys: a guide of useful concepts and tools, in Nanoalloys. Engineering Materials, edited by D. Alloyeau, C. Mottet, and C. Ricolleau (Springer, London, 2012), p. 159.

[19] P. M. Larsen, A. R. Kalidindi, S. Schmidt, and C. A. Schuh, Alloy design as an inverse problem of cluster expansion models, Acta Mater. 139, 254 (2017).

[20] N. A. Zarkevich, T. L. Tan, L.-L. Wang, and D. D. Johnson, Low-Energy antiphase boundaries, degenerate superstructures, and phase stability in frustrated fcc Ising model and Ag-Au alloys, Phys. Rev. B 77, 144208 (2008).

[21] D. Paudyal, T. Saha-Dasgupta, and A. Mookerjee, Phase stability analysis in Fe-Pt and Co-Pt alloy systems: An augmented space study, J. Phys. Condens. Matter 16, 7247 (2004).

[22] J. Teeriniemi, P. Taskinen, and K. Laasonen, First-Principles investigation of the $\mathrm{Cu}-\mathrm{Ni}, \mathrm{Cu}-\mathrm{Pd}$, and $\mathrm{Ni}-\mathrm{Pd}$ binary alloy systems, Intermetallics 57, 41 (2015).

[23] S. He, P. Peng, O. I. Gorbatov, and A. V. Ruban, Effective interactions and atomic ordering in Ni-rich Ni-Re alloys, Phys. Rev. B 94, 024111 (2016).

[24] I. Al-Lehyani, M. Widom, Y. Wang, N. Moghadam, G. M. Stocks, and J. A. Moriarty, Transition-metal interactions in aluminum-rich intermetallics, Phys. Rev. B 64, 075109 (2001).

[25] A. V. Ruban, S. Shallcross, S. I. Simak, and H. L. Skriver, Atomic and magnetic configurational energetics by the generalized perturbation method, Phys. Rev. B 70, 125115 (2004).

[26] M. Fèvre, J.-M. Sanchez, J. R. Stewart, J.-S. Mérot, F. Fossard, Y. Le Bouar, K. Tanaka, H. Numakura, G. Schmerber, and V. Pierron-Bohnes, Investigations of the Co-Pt alloy phase diagram with neutron diffuse scattering, inverse cluster variation method, and Monte Carlo simulations, Phys. Rev. B 102, 134114 (2020).

[27] F. Körmann, A. V. Ruban, and M. H. F. Sluiter, Long-ranged interactions in bcc NbMoTaW high-entropy alloys, Mater. Res. Lett. 5, 35 (2017).

[28] X. Xu and H. Jiang, Cluster expansion based configurational averaging approach to bandgaps of semiconductor alloys, J. Chem. Phys. 150, 034102 (2019).

[29] A. R. Natarajan and A. Van der Ven, Linking electronic structure calculations to generalized stacking fault energies in multicomponent alloys, Npj Comput. Mater. 6, 80 (2020).

[30] G. Ceder, A. F. Kohan, M. K. Aydinol, P. D. Tepesch, and A. Ven, Thermodynamics of oxides with substitutional disorder: a microscopic model and evaluation of important energy contributions, J. Am. Ceram. Soc. 81, 517 (2005).

[31] N. L. Allan, G. D. Barrera, M. Y. Lavrentiev, C. L. Freeman, I. T. Todorov, and J. A. Purton, Beyond the point defect limit: simulation methods for solid solutions and highly disordered systems, Comput. Mater. Sci. 36, 42 (2006).

[32] X. Liu, V. L. Vinograd, X. Lu, E. V Leonenko, N. N. Eremin, R. Wang, and B. Winkler, Thermodynamics of mixing in an isostructural solid solution: simulation methodologies and 
application to the rutile-cassiterite system, Am. Mineral. 101, 1197 (2016).

[33] H. Ji, A. Urban, D. A. Kitchaev, D. H. Kwon, N. Artrith, C. Ophus, W. Huang, Z. Cai, T. Shi, J. C. Kim, H. Kim, and G. Ceder, Hidden structural and chemical order controls lithium transport in cation-disordered oxides for rechargeable batteries, Nat. Commun. 10, 592 (2019).

[34] A. van de Walle, M. Asta, and G. Ceder, The alloy theoretic automated toolkit: A user guide, Calphad 26, 539 (2002).

[35] J. H. Chang, D. Kleiven, M. Melander, J. Akola, J. M. GarciaLastra, and T. Vegge, CLEASE: A versatile and user-friendly implementation of cluster expansion method, J. Phys. Condens. Matter 31, 325901 (2019).

[36] M. Asta, C. Wolverton, D. de Fontaine, and H. Dreyssé, Effective cluster interactions from cluster-variation formalism. I, Phys. Rev. B 44, 4907 (1991).

[37] C. Wolverton, M. Asta, H. Dreyssé, and D. de Fontaine, Effective cluster interactions from cluster-variation formalism. II, Phys. Rev. B 44, 4914 (1991).

[38] A. Gonis, P. P. Singh, P. E. A. Turchi, and X. G. Zhang, Use of the Ising model in the study of substitutional alloys, Phys. Rev. B 51, 2122 (1995).

[39] A. V. Ruban and I. A. Abrikosov, Configurational thermodynamics of alloys from first principles: Effective cluster interactions, Rep. Prog. Phys. 71, 046501 (2008).

[40] A. H. Nguyen, C. W. Rosenbrock, C. S. Reese, and G. L. W. Hart, Robustness of the cluster expansion: Assessing the roles of relaxation and numerical error, Phys. Rev. B 96, 014107 (2017).

[41] N. A. Zarkevich and D. D. Johnson, Reliable First-Principles Alloy Thermodynamics via Truncated Cluster Expansions, Phys. Rev. Lett. 92, 255702 (2004).

[42] M. Stone, Cross-validatory choice and assessment of statistical predictions, J. R. Stat. Soc. Series B Stat. Methodol. 36, 111 (1974).

[43] D. M. Allen, The relationship between variable selection and data agumentation [sic] and a method for prediction, Technometrics 16, 125 (1974).

[44] J. M. Sanchez, Foundations and practical implementations of the cluster expansion, J. Phase Equilibria Diffus. 38, 238 (2017).

[45] J. M. Sanchez, Renormalized interactions in truncated cluster expansions, Phys. Rev. B 99, 134206 (2019).

[46] F. Berthier, J. Creuze, and B. Legrand, Effective site-energy model: a thermodynamic approach applied to size-mismatched alloys, Phys. Rev. B 95, 224102 (2017).

[47] F. Berthier, J. Creuze, T. Gabard, B. Legrand, M. C. Marinica, and C. Mottet, Order-disorder or phase-separation transition: analysis of the Au-Pd system by the effective site energy model, Phys. Rev. B 99, 014108 (2019).

[48] F. Berthier and B. Legrand, Analysis of Au-Pd driving forces via the effective site energy model: LRO, antisites and enthalpy of permutation, J. Phys. Condens. Matter 32, 354001 (2020).

[49] H. Lukas, S. G. Fries, and B. Sundman, Computational Thermodynamics, Vols. 978-0-521- (Cambridge University Press, Cambridge, 2007).

[50] M. Cottura and E. Clouet, Solubility in $\mathrm{Zr}-\mathrm{Nb}$ alloys from firstprinciples, Acta Mater. 144, 21 (2018).

[51] C. Goyhenex, H. Bulou, J.-P. Deville, and G. Tréglia, $\mathrm{Pt} / \mathrm{Co}(0001)$ superstructures in the submonolayer range: $\mathrm{A}$ tight-binding quenched-molecular-dynamics study, Phys. Rev. B 60, 2781 (1999).

[52] G. Rossi, R. Ferrando, and C. Mottet, Structure and chemical ordering in CoPt nanoalloys, Faraday Discuss. 138, 193 (2008).

[53] Z. Yang, L. Shi, and J. Ni, A computational study of kinetic phase diagrams for CoPt alloy films during epitaxial growth, Thin Solid Films 518, 4860 (2010).

[54] A. Lopes, G. Tréglia, C. Mottet, and B. Legrand, Ordering and surface segregation in $\mathrm{Co}_{1-c} \mathrm{Pt}_{c}$ nanoparticles: A theoretical study from surface alloys to nanoalloys, Phys. Rev. B 91, 035407 (2015).

[55] A. Front, B. Legrand, G. Tréglia, and C. Mottet, Bidimensional phases in Co-Pt surface alloys: A theoretical study of ordering and surface segregation, Surf. Sci. 679, 128 (2019).

[56] D. D. Johnson, A. V. Smirnov, J. B. Staunton, F. J. Pinski, and W. A. Shelton, Temperature-induced configurational excitations for predicting thermodynamic and mechanical properties of alloys, Phys. Rev. B 62, R11917 (2000).

[57] J. M. Sanchez, Cluster expansion and the configurational theory of alloys, Phys. Rev. B 81, 224202 (2010).

[58] A. Finel, M. Barrachin, R. Caudron, and A. Francois, Effective pairwise interactions in $\mathrm{Ni}_{3} \mathrm{~V}$, in Metallic Alloys: Experimental and Theoretical Perspectives (Springer Netherlands, Dordrecht, 1994), pp. 215-224.

[59] E. Walter, Y. Lecourtier, J.-Y. Kao, and J. Happel, Identifiability and distinguishability testing for linear models in heterogeneous catalysis, Chem. Eng. Commun. 83, 157 (1989).

[60] E. Walter and L. Pronzato, On the identifiability and distinguishability of nonlinear parametric models, Math. Comput. Simul. 42, 125 (1996).

[61] F. Berthier, J.-P. Diard, L. Pronzato, and E. Walter, Identifiability and distinguishability concepts in electrochemistry, Automatica 32, 973 (1996). 